\title{
Cascade Michael-Aldol reaction: efficient annulation of sulfonamide chalcones into novel cyclohexenones under solvent-free conditions
}

\author{
Nikita R. Agrawal, ${ }^{\ddagger}$ Sandeep P. Bahekar, ${ }^{\star}$ Abhijeet R. Agrawal ${ }^{\dagger}$, Prashant B. Sarode, and \\ Hemant S. Chandak* \\ Department of Chemistry, G. S. Science, Arts and Commerce College, Khamgaon, 444303 India. \\ ${ }^{\dagger}$ Present address: Department of Chemical Sciences, IISER-Kolkata,Mohanpur, 741246, India \\ Equal contribution from both the authors \\ E-mail: chemants@gmail.com
}

DOI: $\underline{\text { http://dx.doi.org/10.3998/ark.5550190.p009.488 }}$

\begin{abstract}
A simple, convenient and efficient synthesis of novel sulfonamide cyclohexenones from differently substituted sulfonamide chalcones has been developed. Syntheses of cyclohexenones have been achieved via cascade Michael-Aldol reaction under solvent free condition. This process features mild and solvent-free synthesis of the titled compounds with high yields (18 examples, up to $95 \%$ yield). The synthesized scaffold is a promising intermediate for the further transformation into various heterocyclic compounds.
\end{abstract}

Keywords: Cascade Michael-Aldol reaction, solvent-free synthesis, sulfonamide cyclohexenones, sulfonamide chalcones

\section{Introduction}

Development of clean synthetic methodologies with least environmental damage is the primary requirement of sustainable chemical process. Nowadays, large scale usage of organic solvents instigate serious environmental threat. ${ }^{1,2}$ To minimize damage to the environment due to waste stream of organic solvents, synthetic chemists are under constant pressure of developing synthetic strategies which includes use of more benign solvents like water, supercritical $\mathrm{CO}_{2}$ and ionic liquids. ${ }^{1}$ It has been said that 'the best solvent is no solvent'. ${ }^{1}$ Solvent-free reactions obviously reduce pollution, and bring down handling costs due to simplification of experimental procedure, work up technique and thereby saving in labor. Reactions on solid support like clays, ${ }^{3}$ zeolites, ${ }^{4}$ silica, ${ }^{5,6}$ alumina. $^{7}$ using conventional heating ${ }^{8}$ or irradiation with microwave ${ }^{9,10}$ or ultrasound $^{11}$ continue to witness exponential growth in the recent times. However, these are not supposed to be 
a 'true solvent-free' reaction as solvents are often used to pre-adsorb the substrate on the solid support. ${ }^{12}$

Sulfonamide chalcones identified as chemotherapeutic agents, possesses potent biological properties, ${ }^{13,14}$ provides a versatile platform for the synthesis of bioactive scaffolds such as cyanopyridines, isoxazoles, pyrazoles and pyrimidin-2-thiones. ${ }^{15}, 16$ Cyclohexenones, being known to display important biological activities such as anticancer agents ${ }^{17}$ and pheromones. ${ }^{18-20}$ In addition, cyclohexenones have been widely found as a key structural skeleton in natural products and pharmaceuticals like saudin, ${ }^{21}$ antroquinonol, ${ }^{22}$ gabosines,${ }^{23}$ etc. A number of synthetic strategies have been reported for the construction of cyclohexenone skeleton which includes Hagemann condensation and Knoevenagel condensation, ${ }^{24-26}$ reductive synthesis via cyclization of heavily functionalized dihydropyrines, ${ }^{27}$ and Au-catalyzed cyclisation of alkenefunctionalized propargyl acetate followed by base treatment. ${ }^{28}$ Robinson annulation of chalcone motif promoted by various catalysts such as lanthanide complex, ${ }^{29}$ Rhodium, ${ }^{30} \mathrm{C}-200,{ }^{31,32}$ etc has also been documented. Specifically, addition of $\beta$-keto ester to chalcone moiety has also been accessed in the presence of base like sodium ethoxide, ${ }^{33}$ potassium carbonate under MW irradiation. ${ }^{34}$ Despite the progress, till date, there is only one report of transformation of sulfonamide chalcones to cyclohexenone skeleton. El-Sharief et al. reported transformation of sulfonamide chalcones to cyclohexenone skeleton using piperidine as a base and butanol as a solvent. ${ }^{35}$

In virtue of extending wide arena of this structurally interesting sulfonamide chalcones and cyclohexenone, herein we report annulations of sulfonamide chalcones with ethyl acetoacetate via cascade Michael-Aldol reaction in presence of anhydrous $\mathrm{K}_{2} \mathrm{CO}_{3}$ under solvent-free condition (Scheme 2).

\section{Results and Discussion}

Aiming to construct cyclohexenone scaffolds (7a-7r), the precursor sulfonamide chalcones 5 were prepared by Claisen-Schmidt condensation of ketone $\mathbf{3}$ and differently substituted aldehydes $\mathbf{4}$ (Scheme 1). Ketone 3, in turn was prepared easily by condensation of aryl/methane sulfonyl chloride $\mathbf{1}$ and 4 -aminoacetophenone $\mathbf{2}$ in presence of triethyl amine as a base. ${ }^{36}$ 
<smiles>[R1]S(=O)(=O)Cl</smiles>

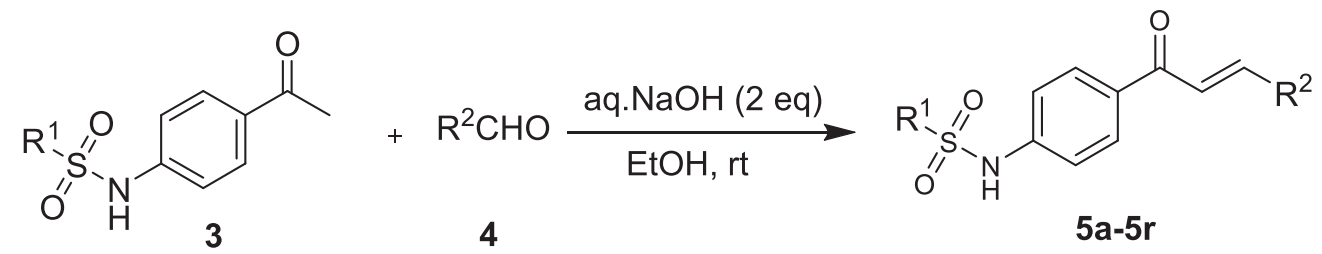

Scheme 1. Synthesis of sulfonamide chalcones (5a-5r).

The concluding step was the annulation of 5a-5r into cyclohexenones possessing sulfonamide unit (7a-7r) via cascade Michael-Aldol reaction. Anhydrous $\mathrm{K}_{2} \mathrm{CO}_{3}$ catalyzed annulation reaction was achieved from sulfonamide chalcones (5a-5r) with ethylacetoacetate at $80{ }^{\circ} \mathrm{C}$ under solventfree condition. When tested the effect of temperature on the proposed reaction, low temperature i.e. $60^{\circ} \mathrm{C}$ took longer time for completion with moderate yields while higher temperature i.e. 100 ${ }^{\circ} \mathrm{C}$ did not boost the reaction rate. After completion of reaction (TLC monitoring), simple extraction from ethanol at reaction temperature offers effortless isolation of pure product with better yields (Scheme 2).<smiles>[R]C=CC(=O)c1ccc(N[SH]([R1])(=O)O[R15])cc1</smiles><smiles>[R]C1CC(c2ccc(NS([R4])(=O)=O)cc2)=CC(=O)C1C(=O)OCC</smiles>

Scheme 2. Synthesis of sulfonamide cyclohexenone derivatives (7a-7r).

Proving the generality of the aforesaid protocol, an array of varied substituted sulfonamide cyclohexenones $7 \mathbf{a}-7 \mathbf{r}$ was synthesized by varying the substitution on the $3^{\text {rd }}$ and $5^{\text {th }}$ position of cyclohexenone ring and the outputs are summarized in Table 1. It can be contemplated that the substitution at phenyl ring of $\mathrm{R}^{2}$ of sulfonamide chalcone profoundly affects the speed of reaction. Reactions with electron-withdrawing substituent at phenyl ring of $\mathrm{R}^{2}$ were found to be more facile than that with electron-donating substituent (Table 1). It might be due to the increase in the electrophilicity of $\beta$-carbon of chalcone which thereon facilitates the nucleophilic attack of ethyl acetoacetate resulting into 1,4 addition which subsequently cyclizes to afford substituted sulfonamide cyclohexenones. Substitution at $\mathrm{R}^{1}$ of phenylsulfonamido moiety shows little or no 
effect on the speed and yield of reaction and reaction proceeds smoothly with better yields (Table 1, entry 7l-7r). Overall yields of the synthesized compounds 7a-7r were found to be excellent.

Table 1. Physical data of sulfonamide cyclohexenones (7a-7r)

\begin{tabular}{|c|c|c|c|c|c|c|}
\hline Entry & $\mathbf{R}^{1}$ & $\mathbf{R}^{2}$ & Product & $\begin{array}{l}\text { Time } \\
\text { (in } \\
\text { min) }\end{array}$ & $\begin{array}{l}\text { Yield } \\
(\%)\end{array}$ & $\begin{array}{l}\mathrm{Mp} \\
\left({ }^{\circ} \mathrm{C}\right)\end{array}$ \\
\hline $7 \mathbf{a}$ & $-\mathrm{Ph}$ & $-\mathrm{Ph}$ & & 60 & 91 & $\begin{array}{l}180- \\
181\end{array}$ \\
\hline $7 b$ & $-\mathrm{Ph}$ & $-4-\mathrm{Cl}-\mathrm{C}_{6} \mathrm{H}_{4}$ & & 50 & 80 & $\begin{array}{l}235- \\
237\end{array}$ \\
\hline $7 c$ & $-\mathrm{Ph}$ & $-4-\mathrm{F}-\mathrm{C}_{6} \mathrm{H}_{4}$ & & 50 & 88 & $\begin{array}{l}254- \\
255\end{array}$ \\
\hline $7 d$ & $-\mathrm{Ph}$ & $-4-\mathrm{Br}-\mathrm{C}_{6} \mathrm{H}_{4}$ & & 50 & 80 & $\begin{array}{l}270- \\
272\end{array}$ \\
\hline $7 e$ & $-\mathrm{Ph}$ & $\begin{array}{l}-4- \\
\mathrm{CH}\left(\mathrm{CH}_{3}\right)_{2}- \\
\mathrm{C}_{6} \mathrm{H}_{4}\end{array}$ & & 70 & 95 & $\begin{array}{l}199- \\
200\end{array}$ \\
\hline $7 f$ & $-\mathrm{Ph}$ & -2-furyl & & 60 & 72 & $\begin{array}{l}196- \\
198\end{array}$ \\
\hline $7 \mathrm{~g}$ & $-\mathrm{Ph}$ & $\begin{array}{l}-3,4,5- \\
\left(\mathrm{OCH}_{3}\right)_{3-} \\
\mathrm{C}_{6} \mathrm{H}_{2}\end{array}$ & & 70 & 80 & $\begin{array}{l}241- \\
243\end{array}$ \\
\hline
\end{tabular}


Table 1 (continued)

\begin{tabular}{|c|c|c|c|c|c|c|}
\hline Entry & $\mathbf{R}^{1}$ & $\mathbf{R}^{2}$ & Product & $\begin{array}{l}\text { Time } \\
\text { (in } \\
\text { min) }\end{array}$ & $\begin{array}{l}\text { Yield } \\
(\%)\end{array}$ & $\begin{array}{l}\text { Мp } \\
\left({ }^{\circ} \mathrm{C}\right)\end{array}$ \\
\hline $7 \mathrm{~h}$ & $-\mathrm{Ph}$ & $-2-\mathrm{Cl}-\mathrm{C}_{6} \mathrm{H}_{4}$ & & 50 & 81 & $\begin{array}{l}210- \\
211\end{array}$ \\
\hline $7 \mathbf{i}$ & $-\mathrm{Ph}$ & $-2-\mathrm{Br}-\mathrm{C}_{6} \mathrm{H}_{4}$ & & 50 & 80 & $\begin{array}{l}224- \\
226\end{array}$ \\
\hline $7 \mathbf{j}$ & $-\mathrm{Ph}$ & $\begin{array}{l}-4-\mathrm{CH}_{3}- \\
\mathrm{C}_{6} \mathrm{H}_{4}\end{array}$ & & 70 & 89 & $\begin{array}{l}193- \\
194\end{array}$ \\
\hline $7 \mathbf{k}$ & $-\mathrm{Ph}$ & $-3-\mathrm{Cl}-\mathrm{C}_{6} \mathrm{H}_{4}$ & & 50 & 90 & $\begin{array}{l}236- \\
237\end{array}$ \\
\hline 71 & $\begin{array}{l}-4- \\
\mathrm{CH}_{3-} \\
\mathrm{C}_{6} \mathrm{H}_{4}\end{array}$ & $-\mathrm{Ph}$ & & 60 & 90 & $\begin{array}{l}180- \\
181\end{array}$ \\
\hline $7 \mathrm{~m}$ & $\begin{array}{l}-4- \\
\mathrm{CH}_{3-} \\
\mathrm{C}_{6} \mathrm{H}_{4}\end{array}$ & $\begin{array}{l}-4- \\
\mathrm{CH}\left(\mathrm{CH}_{3}\right)_{2}- \\
\mathrm{C}_{6} \mathrm{H}_{4}\end{array}$ & & 70 & 95 & $\begin{array}{l}211- \\
212\end{array}$ \\
\hline $7 n$ & $\begin{array}{l}-4- \\
\mathrm{CH}_{3-} \\
\mathrm{C}_{6} \mathrm{H}_{4}\end{array}$ & $\begin{array}{l}-3,4,5- \\
\left(\mathrm{OCH}_{3}\right)_{3-} \\
\mathrm{C}_{6} \mathrm{H}_{2}\end{array}$ & & 70 & 93 & $\begin{array}{l}256- \\
257\end{array}$ \\
\hline
\end{tabular}


Table 1 (continued)

\begin{tabular}{|c|c|c|c|c|c|c|}
\hline Entry & $\mathbf{R}^{1}$ & $\mathbf{R}^{2}$ & Product & $\begin{array}{l}\text { Time } \\
\text { (in } \\
\text { min) }\end{array}$ & $\begin{array}{l}\text { Yield } \\
(\%)\end{array}$ & $\begin{array}{l}\mathrm{Mp} \\
\left({ }^{\circ} \mathrm{C}\right)\end{array}$ \\
\hline 70 & $\begin{array}{l}-4- \\
\mathrm{CH}_{3-} \\
\mathrm{C}_{6} \mathrm{H}_{4}\end{array}$ & $\begin{array}{l}-4-\mathrm{CH}_{3}- \\
\mathrm{C}_{6} \mathrm{H}_{4}\end{array}$ & & 70 & 80 & $\begin{array}{l}201- \\
202\end{array}$ \\
\hline $7 p$ & $\begin{array}{l}-4- \\
\mathrm{CH}_{3-} \\
\mathrm{C}_{6} \mathrm{H}_{4}\end{array}$ & $-3-\mathrm{Cl}-\mathrm{C}_{6} \mathrm{H}_{4}$ & & 50 & 91 & $\begin{array}{l}240- \\
241\end{array}$ \\
\hline $7 q$ & $-\mathrm{CH}_{3}$ & $-4-\mathrm{Cl}-\mathrm{C}_{6} \mathrm{H}_{4}$ & & 50 & 92 & $\begin{array}{l}181- \\
182\end{array}$ \\
\hline $7 r$ & $-\mathrm{CH}_{3}$ & $\begin{array}{l}-3,4,5- \\
\left(\mathrm{OCH}_{3}\right)_{3-} \\
\mathrm{C}_{6} \mathrm{H}_{2}\end{array}$ & & 70 & 84 & $\begin{array}{l}194- \\
195\end{array}$ \\
\hline
\end{tabular}

Structures of newly synthesized sulfonamide cyclohexenones were characterized by $\mathrm{IR},{ }^{1} \mathrm{H}$ NMR, ${ }^{13} \mathrm{C}$ NMR, mass spectrometric and elemental analysis. IR spectrum clearly indicates two absorption peaks for ketone and ester carbonyl in the range of $1625-1658 \mathrm{~cm}^{-1}$ and $1716-1738 \mathrm{~cm}^{-}$ ${ }^{1}$ respectively. N-H stretch at absorption band was observed around $3200 \mathrm{~cm}^{-1}$ while symmetric and asymmetric stretch of $-\mathrm{SO}_{2} \mathrm{NH}$ group was observed around 1080 and $1350 \mathrm{~cm}^{-1}$ respectively. In ${ }^{1} \mathrm{H}$ spectrum, there was a singlet around $\delta 6.3$ for the $\mathbf{H}_{5}$ proton of $\mathrm{C}_{2}$-carbon of cyclohexenone ring. Methyl group of ethoxycarbonyl moiety resonated around $\delta 0.9$, while quartet of corresponding methylene group of ethoxycarbonyl group merged with $\mathbf{H}_{4}$ and observed as complex multiplets around $\delta 3.8$. The remainder protons $\mathbf{H}_{\mathbf{1}}$ and $\mathbf{H}_{\mathbf{2}}$ resonated around $\delta$ 2.9, while $\mathbf{H}_{3}$ observed as multiplet around $\delta 3.5$ (Figure 1). ${ }^{13} \mathrm{C}$ NMR spectra showed requisite number of distinct resonances in agreement with the destined structure. ESI-MS of the compounds showed molecular ion peaks at their respective $\mathrm{m} / \mathrm{e}$ value. 


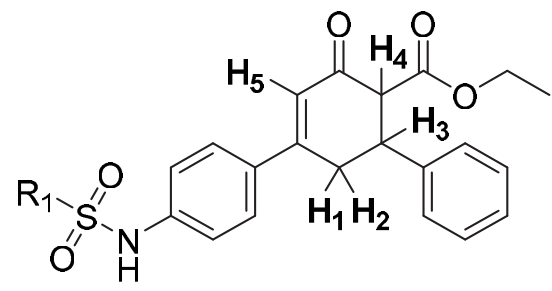

Figure 1. Cyclohexenone scaffold showing different types of proton.

\section{Conclusions}

We have illustrated an efficient synthesis of novel sulfonamide cyclohexenones via cascade Michael-Aldol reaction in the presence of anhydrous $\mathrm{K}_{2} \mathrm{CO}_{3}$ under solvent-free condition. The demonstrated protocol is wide in scope with the respect to the change in substituent at both the phenyl rings of the precursor chalcone. The fascinating merits of present protocol are the environmentally friendly conditions, simplicity of reaction, reasonable reaction times, very good yields and simple workup procedure. The synthesized scaffolds are the promising intermediate for further transformation into heterocycles, thus present research work likely to open gateway for the future scope for synthesis of the biologically potential heterocyclic skeletons.

\section{Experimental Section}

General. All solvents and chemicals were obtained commercially and were used as received. Melting points were determined in an open capillary and are uncorrected. IR spectra were recorded using a Bruker instrument. NMR spectra were taken with a Bruker Avance II at $400 \mathrm{MHz}$ / Bruker DMX spectrometer at $400 \mathrm{MHz}\left({ }^{1} \mathrm{H}\right)$ and $100 \mathrm{MHz}\left({ }^{13} \mathrm{C}\right)$ using DMSO- $d_{6}$ as the solvent. All chemical shifts are reported in ppm and are referenced to tetramethylsilane using residual ${ }^{1} \mathrm{H}$ signals of the deuterated solvents as internal standards. Electron spray ionization mass spectra were recorded on Bruker microTOFQ spectrometer. Elemental analyses $(\mathrm{C}, \mathrm{H}, \mathrm{N})$ were obtained using Carlo Erba 1108 analyzer.

\section{General procedure for the synthesis of sulfonamide ketone (3)}

Aryl/methane sulphonyl chloride $1(10 \mathrm{mmol})$, 4-aminoacetophenone $\mathbf{2}(10 \mathrm{mmol})$ and triethyl amine $(10 \mathrm{mmol})$ in ethanol $(15 \mathrm{ml})$ were refluxed until the TLC showed the complete consumption of starting material 2 i.e. for $4 \mathrm{hr}$. After completion of reaction, contents were cooled and poured into dilute $\mathrm{HCl}$. The solid obtained was filtered, washed with $2 \% \mathrm{NaHCO}_{3}$ and again with water to get crude product which was then recystallized with ethanol- water (1:1) to get pure ketone 3. 
$\mathrm{N}$-(4-acetylphenyl)benzenesulfonamide (3a). Yellow crystal, yield $87 \%$, mp 126-127 $\mathrm{C}$ (lit. $\left.127-130^{\circ} \mathrm{C}\right)^{37}$.

$\mathrm{N}$-(4-acetylphenyl)-4-methylbenzenesulfonamide (3b). Yellow crystal, yield $90 \%$, mp 203$204^{\circ} \mathrm{C}$ (lit. $\left.204-206^{\circ} \mathrm{C}\right)^{38}$.

$\mathrm{N}$-(4-acetylphenyl)methanesulfonamide (3c). Yellow crystal, yield $85 \%$, mp $157-158{ }^{\circ} \mathrm{C}$ (lit. $\left.156-156.5^{\circ} \mathrm{C}\right)^{39}$.

\section{General procedure for the synthesis of sulfonamide chalcones (5a-5r)}

To the solution of $\mathbf{3}(5 \mathrm{mmol})$ and aldehydes $4(5 \mathrm{mmol})$ in ethanol $(10 \mathrm{ml})$, aq. $\mathrm{NaOH}(10 \mathrm{mmol})$ was added drop-wise with constant stirring for 30 min during which a yellow cake was formed. It was then kept overnight at room temperature. The solid cake so obtained was acidified with dilute $\mathrm{HCl}$. The solid obtained was filtered, washed with $2 \% \mathrm{NaHCO}_{3}$ and again with water to get crude product which was then recrystallized with ethanol to get yellow crystals of (5a-5r).

$\mathrm{N}$-(4-cinnamoylphenyl)benzenesulfonamide (5a). White solid, yield $83 \%$, mp $183-185^{\circ} \mathrm{C}$ (lit. $\left.180^{\circ} \mathrm{C}\right),{ }^{40}$ Rf 0.5 (30\% EtOAc:Hexane); IR $\left(v_{\max }, \mathrm{cm}^{-1}\right): 3219$ (N-H str), 3057 (Ar C-H str), 1651 (C=O str), 1336 (asymm. $\mathrm{S}=\mathrm{O}$ str), 1165 (symm. $\mathrm{S}=\mathrm{O}$ str); ${ }^{1} \mathrm{H}$ NMR (400MHz, DMSO-d $)$ ): 10.91 $\left(\mathrm{s}, 1 \mathrm{H}, \mathrm{SO}_{2} \mathrm{NH}\right), 8.04-8.02(\mathrm{~m}, 2 \mathrm{H}, \mathrm{Ar}-\mathrm{H}), 7.86-7.82(\mathrm{~m}, 5 \mathrm{H}, \mathrm{Ar}-\mathrm{H}), 7.70-7.56(\mathrm{~m}, 4 \mathrm{H}, \mathrm{Ar}-$ $\mathrm{H}+\mathrm{CH}=\mathrm{CH}), 7.44-7.43(\mathrm{~m}, 3 \mathrm{H}, \mathrm{Ar}-\mathrm{H}), 7.27-7.25(\mathrm{~m}, 2 \mathrm{H}, \mathrm{Ar}-\mathrm{H}) ;{ }^{13} \mathrm{C}$ NMR $\left(125 \mathrm{MHz}, \mathrm{CDCl}_{3}\right) \delta$ $188.87,144.89,140.75,138.86,134.78,134.44,133.44,130.60,130.16,129.26,128.95,128.43$, 127.19, 121.50, 119.33; MS (ESI): $m / z[\mathrm{M}+\mathrm{H}]^{+}$calcd for $\mathrm{C}_{21} \mathrm{H}_{18} \mathrm{NO}_{3} \mathrm{~S} \mathrm{~m} / \mathrm{z}$ 364.0929, observed $364.1125[\mathrm{M}+\mathrm{H}]^{+}$.

$\mathrm{N}$-[4-(3-(4-chlorophenyl)-acryloyl)-phenyl]-benzenesulfonamide (5b). Yellow solid, yield 74\%, mp 200-202 ${ }^{\circ}$, Rf 0.4 (30\% EtOAc:Hexane); IR ( $\left.v_{\max }, \mathrm{cm}^{-1}\right): 3280$ (N-H str), 3064 (Ar C-H str), 1651 ( $\mathrm{C}=\mathrm{O}$ str), 1301 (asymm. $\mathrm{S}=\mathrm{O}$ str), 1157 (symm. $\mathrm{S}=\mathrm{O}$ str); ${ }^{1} \mathrm{H}$ NMR (400MHz, DMSO$\left.d_{6}\right): 10.96\left(\mathrm{~s}, 1 \mathrm{H}, \mathrm{SO}_{2} \mathrm{NH}\right), 8.07-8.05(\mathrm{~m}, 2 \mathrm{H}, \mathrm{Ar}-\mathrm{H}), 7.90-7.85$ (m, 5H, Ar-H), 7.69-7.56 (m, 4H, $\mathrm{Ar}-\mathrm{H}+\mathrm{CH}=\mathrm{CH}), 7.52-7.50(\mathrm{~m}, 2 \mathrm{H}, \mathrm{Ar}-\mathrm{H}), 7.29-7.25(\mathrm{~m}, 2 \mathrm{H}, \mathrm{Ar}-\mathrm{H}) ;{ }^{13} \mathrm{C} \mathrm{NMR}\left(125 \mathrm{MHz}, \mathrm{CDCl}_{3}\right)$ $\delta 188.78,140.97,140.65,138.83,135.47,134.46,133.19,131.20,130.85,130.31,129.27,127.33$, 127.18, 127.06, 124.36, 119.35; MS (ESI): $m / z[\mathrm{M}+\mathrm{H}]^{+}$calcd for $\mathrm{C}_{21} \mathrm{H}_{17} \mathrm{ClNO}_{3} \mathrm{~S} \mathrm{~m} / \mathrm{z} 398.0539$, observed $398.0678[\mathrm{M}+\mathrm{H}]^{+}$.

$\mathrm{N}$-[4-(3-(4-fluorophenyl)-acryloyl)-phenyl]-benzenesulfonamide (5c). Yellow solid, yield 64\%, mp 165-167 C, Rf 0.4 (30\% EtOAc:Hexane); IR ( $\left.v_{\max }, \mathrm{cm}^{-1}\right): 3273$ (N-H str), 3066 (Ar C-H str), 1654 (C=O str), 1340 (asymm. $\mathrm{S}=\mathrm{O}$ str), 1157 (symm. $\mathrm{S}=\mathrm{O}$ str); ${ }^{1} \mathrm{H}$ NMR (400MHz, DMSO$\left.d_{6}\right): 10.94\left(\mathrm{~s}, 1 \mathrm{H}, \mathrm{SO}_{2} \mathrm{NH}\right), 8.06-8.04(\mathrm{~m}, 2 \mathrm{H}, \mathrm{Ar}-\mathrm{H}), 7.95-7.91(\mathrm{~m}, 2 \mathrm{H}, \mathrm{Ar}-\mathrm{H}), 7.87-7.80(\mathrm{~m}, 4 \mathrm{H}$, $\mathrm{Ar}-\mathrm{H}+\mathrm{CH}=\mathrm{CH}), 7.70-7.56$ (m, 4H, Ar-H), 7.31-7.24 (m, 2H, Ar-H); ${ }^{13} \mathrm{C}$ NMR (125 MHz, $\left.\mathrm{CDCl}_{3}\right)$ $\delta 188.84,165.40,163.07,143.75,143.72,133.65,130.57,130.48,130.35,129.45,127.32,121.24$, 119.43, 116.43, 116.18; MS (ESI): $m / z[\mathrm{M}+\mathrm{H}]^{+}$calcd for $\mathrm{C}_{21} \mathrm{H}_{17} \mathrm{FNO}_{3} \mathrm{~S} \mathrm{~m} / \mathrm{z} 382.0835$, observed $382.0983[\mathrm{M}+\mathrm{H}]^{+}$. 
$\mathrm{N}$-[4-(3-(4-bromophenyl)-acryloyl)-phenyl]-benzenesulfonamide (5d). Yellow solid, yield 71\%, mp 205-207 C, Rf 0.4 (30\% EtOAc:Hexane); IR ( $\left.v_{\max }, \mathrm{cm}^{-1}\right): 3278$ (N-H str), 3055 (Ar C-H str), 1651 ( $\mathrm{C}=\mathrm{C}$ str), 1300 (asymm. $\mathrm{S}=\mathrm{O}$ str), 1157 (symm. $\mathrm{S}=\mathrm{O}$ str); ${ }^{1} \mathrm{H}$ NMR (400MHz, DMSO$\left.d_{6}\right): 10.95\left(\mathrm{~s}, 1 \mathrm{H}, \mathrm{SO}_{2} \mathrm{NH}\right), 8.06-8.04(\mathrm{~m}, 2 \mathrm{H}, \mathrm{Ar}-\mathrm{H}), 7.91-7.80(\mathrm{~m}, 5 \mathrm{H}, \mathrm{Ar}-\mathrm{H}), 7.66-7.58(\mathrm{~m}, 6 \mathrm{H}$, $\mathrm{Ar}-\mathrm{H}+\mathrm{CH}=\mathrm{CH}), 7.26-7.24(\mathrm{~m}, 2 \mathrm{H}, \mathrm{Ar}-\mathrm{H}) ;{ }^{13} \mathrm{C} \mathrm{NMR}\left(125 \mathrm{MHz}, \mathrm{CDCl}_{3}\right) \delta 188.49,143.39,140.84$, 138.88, 134.25, 133.70, 133.45, 132.22, 130.17, 129.75, 129.27, 127.18, 124.88, 121.98, 119.32; MS (ESI): $m / z[\mathrm{M}+\mathrm{H}]^{+}$calcd for $\mathrm{C}_{21} \mathrm{H}_{17} \mathrm{BrNO}_{3} \mathrm{~S} \mathrm{~m} / \mathrm{z} 444.330$, observed $444.0243[\mathrm{M}+\mathrm{H}]^{+}$.

$\mathrm{N}$-(4-(3-(4-isopropylphenyl)acryloyl)phenyl)benzenesulfonamide (5e). Yellow solid, yield 80\%, mp 160-162 ${ }^{\circ}$, Rf 0.4 (30\% EtOAc:Hexane); IR ( $\left.v_{\max }, \mathrm{cm}^{-1}\right): 3203$ (N-H str), 3030 (Ar C-H str), 1649 ( $\mathrm{C}=\mathrm{O}$ str), 1328 (symm. $\mathrm{S}=\mathrm{O}$ str), 1163 (asymm. $\mathrm{S}=\mathrm{O}$ str); ${ }^{1} \mathrm{H}$ NMR (500MHz, DMSO$\left.d_{6}\right)$ : 7.80-7.70 (m, 4H, Ar-H+CH=CH), $7.63(\mathrm{~d}, J 15.6 \mathrm{~Hz}, 1 \mathrm{H}, \mathrm{CH}=\mathrm{CH}), 7.42-7.37$ (m, 3H, ArH), 7.33-7.26 (m, 3H, Ar-H), 7.13-7.07 (m, 4H, Ar-H), 2.78 (septet, J 6.9 Hz, 1H, CH(CH $\left.\mathrm{CH}_{2}\right)_{2}$, $1.11\left(\mathrm{~d}, J 6.9 \mathrm{~Hz}, 6 \mathrm{H}, \mathrm{CH}\left(\mathrm{CH}_{3}\right)_{2}\right) ;{ }^{13} \mathrm{C} \mathrm{NMR}\left(125 \mathrm{MHz}, \mathrm{CDCl}_{3}\right) \delta 189.13,152.07,145.07,140.82$, 138.87, 134.44, 133.38, 132.40, 130.11, 129.23, 128.60, 127.17, 127.07, 120.57, 119.27, 34.11, 23.71; MS (ESI): $m / z[\mathrm{M}+\mathrm{H}]^{+}$calcd for $\mathrm{C}_{24} \mathrm{H}_{24} \mathrm{NO}_{3} \mathrm{~S} \mathrm{~m} / \mathrm{z} 405.1399$, observed $405.6184[\mathrm{M}+\mathrm{H}]^{+}$. $\mathrm{N}$-(4-(3-(furan-2-yl)acryloyl)phenyl)benzenesulfonamide (5f). Yellow solid, yield 73\%, mp 142-144 ${ }^{\circ} \mathrm{C}$, Rf 0.5 (30\% EtOAc:Hexane); IR ( $\left.v_{\max }, \mathrm{cm}^{-1}\right)$ : 3251 (N-H str), 1604 (C=O str), 1331 (asymm. S=O str), 1156 (symm. $\mathrm{S}=\mathrm{O}$ str); ${ }^{1} \mathrm{H}$ NMR (300MHz, DMSO- $d_{6}$ ): 10.93 (s, 1H, NH), 7.97-7.84 (m, 5H, Ar-H), 7.70-7.47 (m, 5H, Ar-H+CH=CH), 7.26-7.24 (m, 2H, Ar-H), 7.06-6.69 $(\mathrm{m}, 2 \mathrm{H}, \mathrm{Ar}-\mathrm{H}) ;{ }^{13} \mathrm{C} \mathrm{NMR}\left(125 \mathrm{MHz}, \mathrm{CDCl}_{3}\right) \delta 188.85,151.30,141.51,133.53,130.32,129.30$, $128.90,127.19,125.18,124.22,119.26,116.51,114.30,110.18$; MS (ESI): $m / z[\mathrm{M}+\mathrm{H}]^{+}$calcd for $\mathrm{C}_{19} \mathrm{H}_{16} \mathrm{NO}_{4} \mathrm{~S} \mathrm{~m} / \mathrm{z} 354.0722$, observed $354.0846[\mathrm{M}+\mathrm{H}]^{+}$.

$\mathrm{N}$-(4-(3-(3,4,5-trimethoxyphenyl)acryloyl)phenyl)benzenesulfonamide (5g). Yellow solid, yield 90\%, mp 190-192 ${ }^{\circ} \mathrm{C}$, Rf 0.3 (30\% EtOAc:Hexane); IR ( $\left.v_{\max }, \mathrm{cm}^{-1}\right): 3219$ (N-H str), 3080 (Ar C-H str), 1649 (C=O str), 1327 (asymm. S=O str), 1157 (symm. S=O str), 1124 (C-O str); ${ }^{1} \mathrm{H} \mathrm{NMR}$ (500 MHz, Chloroform- $d$ ) $\delta 7.94-7.84$ (m, 4H, Ar-H), 7.70 (d, J 14.8 Hz, 1H, CH=CH), $7.58-$ 7.45 (m, 3H, Ar-H), 7.33 (d, J $15.3 \mathrm{~Hz}, 1 \mathrm{H}, \mathrm{CH}=\mathrm{CH}), 7.22$ (s, 2H, Ar-H), $6.89-6.79$ (m, 2H, Ar$\mathrm{H}), 3.91\left(\mathrm{~s}, 9 \mathrm{H}, \mathrm{OCH}_{3}\right) ;{ }^{13} \mathrm{C} \mathrm{NMR}\left(125 \mathrm{MHz}, \mathrm{CDCl}_{3}\right) \delta 188.86,153.49,145.08,140.71,133.42$, $130.14,129.26,127.17,120.84,119.34,105.74,61.00,56.24$; Anal. calcd for $\mathrm{C}_{24} \mathrm{H}_{23} \mathrm{NO}_{6} \mathrm{~S}$ (453.50): C, 63.56; H, 5.11; N, $3.09 \%$. Found: C, 64.38; H, 5.29; N, $3.30 \%$.

$\mathrm{N}$-(4-(3-(2-chlorophenyl)acryloyl)phenyl)benzenesulfonamide (5h). Yellow solid, yield 72\%, mp 169-170 ${ }^{\circ} \mathrm{C}$, Rf 0.6 (30\% EtOAc:Hexane); IR ( $\left.v_{\max }, \mathrm{cm}^{-1}\right)$ : 3211 (N-H str), 3062 (Ar C-H str), 1649 (C=O str), 1332 (asymm. S=O str), 1168 (symm. S=O str); ${ }^{1} \mathrm{H}$ NMR (400MHz, DMSO- $d_{6}$ ): 10.98 (s, $\left.1 \mathrm{H}, \mathrm{SO}_{2} \mathrm{NH}\right), 8.18-8.15(\mathrm{~m}, 1 \mathrm{H}, \mathrm{CH}=\mathrm{CH}), 8.08-8.05(\mathrm{~m}, 2 \mathrm{H}, \mathrm{Ar}-\mathrm{H}), 8.00-7.93(\mathrm{~m}, 2 \mathrm{H}$, Ar-H)), 7.89-7.85 ( m, 2H, ArH) 7.65-7.55 (m, 4H, Ar-H+CH=CH), 7.47- 7.44 (m,2H, ArH), 7.277.25 (m, 2H, Ar-H); ${ }^{13} \mathrm{C}$ NMR $\left(125 \mathrm{MHz}, \mathrm{CDCl}_{3}\right) \delta 188.85,140.90,140.67,138.83,135.47$, 134.13, 133.45, 133.16, 131.20, 130.31, 130.29, 129.27, 127.78, 127.18, 127.06, 124.35, 119.30; MS (ESI): $m / z[\mathrm{M}+\mathrm{H}]^{+}$calcd for $\mathrm{C}_{21} \mathrm{H}_{17} \mathrm{ClNO}_{3} \mathrm{~S} \mathrm{~m} / \mathrm{z} 398.0539$, observed $398.2441[\mathrm{M}+\mathrm{H}]^{+}$.

$\mathrm{N}$-(4-(3-(2-bromophenyl)acryloyl)phenyl)benzenesulfonamide (5i). Yellow solid, yield 82\%, mp 185-186 ${ }^{\circ}$ C, Rf 0.6 (30\% EtOAc:Hexane); IR ( $\left.v_{\max }, \mathrm{cm}^{-1}\right): 3208$ (N-H str), 3066 (Ar C-H str), 
1650 (C=O str), 1338 (asymm. $\mathrm{S}=\mathrm{O}$ str), 1158 (symm. $\mathrm{S}=\mathrm{O}$ str); ${ }^{1} \mathrm{H}$ NMR $\left(500 \mathrm{MHz}, \mathrm{CDCl}_{3}\right) \delta$ $8.11-8.06(\mathrm{~m}, 1 \mathrm{H}, \mathrm{CH}=\mathrm{CH}), 7.94-7.91(\mathrm{~m}, 2 \mathrm{H}, \mathrm{Ar}-\mathrm{H}), 7.89-7.85(\mathrm{~m}, 2 \mathrm{H}, \mathrm{Ar}-\mathrm{H}), 7.70$ (dd, $J$ 7.8, 1.6 Hz, 1H, Ar-H), 7.63 (dd, J 8.0, $1.3 \mathrm{~Hz}, 1 \mathrm{H}, \mathrm{Ar}-\mathrm{H}), 7.59-7.55$ (m, 1H, CH=CH), 7.48 (dd, $J$ 8.5, 7.1 Hz, 2H, Ar-H), $7.38-7.33$ (m, 2H, Ar-H), $7.27-7.20$ (m, 3H, Ar-H); ${ }^{13} \mathrm{C}$ NMR (125 MHz, Chloroform- $d$ ) $\delta$ 188.86, 143.24, 140.91, 138.83, 134.98, 134.10, 133.56, 133.45, 131.35, $130.32,129.27,127.86,127.69,127.19,125.85,124.62,119.31$; Anal. calcd for $\mathrm{C}_{21} \mathrm{H}_{16} \mathrm{BrNO}_{3} \mathrm{~S}$ (442.32): C, 57.02; H, 3.65; N, 3.17\%. Found: C, 57.28; H, 3.59; N, $3.30 \%$.

$\mathrm{N}$-(4-(3-(p-tolyl)acryloyl)phenyl)benzenesulfonamide (5j). Yellow solid, yield 88\%, mp 171$172{ }^{\circ} \mathrm{C}$, Rf 0.52 (30\% EtOAc:Hexane); IR ( $\left.v_{\max }, \mathrm{cm}^{-1}\right)$ : 3206 (N-H str), 3048 (Ar C-H str), 1590 (C=O str), 1345 (asymm. $\mathrm{S}=\mathrm{O}$ str), 1154 (symm. $\mathrm{S}=\mathrm{O}$ str); ${ }^{1} \mathrm{H}$ NMR (500 MHz, $\left.\mathrm{CDCl}_{3}\right) \delta 8.00-$ $7.96(\mathrm{~m}, 2 \mathrm{H}, \mathrm{Ar}-\mathrm{H}+\mathrm{CH}=\mathrm{CH}), 7.94-7.90(\mathrm{~m}, 2 \mathrm{H}, \mathrm{Ar}-\mathrm{H}), 7.83(\mathrm{~d}, J 15.6 \mathrm{~Hz}, 1 \mathrm{H}, \mathrm{CH}=\mathrm{CH}), 7.64$ - 7.46 (m, 7H, Ar-H), 7.29 - 7.26 (m, 3H, Ar-H), 2.45 (s, 3H, $\left.\mathrm{CH}_{3}\right) ;{ }^{13} \mathrm{C} \mathrm{NMR}\left(125 \mathrm{MHz}, \mathrm{CDCl}_{3}\right)$ $\delta 189.00,145.01,141.19,140.67,138.88,134.60,133.41,132.05,130.11,129.70,129.25,128.47$, 127.19, 120.50, 119.36, 21.52; Anal. calcd for $\mathrm{C}_{22} \mathrm{H}_{19} \mathrm{NO}_{3} \mathrm{~S}$ (377.45): C, 70.01; H, 5.07; N, 3.71 \%. Found: C, 69.88; H, 5.29; N, $3.60 \%$.

$\mathrm{N}$-(4-(3-(3-chlorophenyl)acryloyl)phenyl)benzenesulfonamide (5k). Yellow solid, yield 72\%, mp 189-190 ${ }^{\circ} \mathrm{C}$, Rf 0.6 (30\% EtOAc:Hexane); IR ( $\left.v_{\max }, \mathrm{cm}^{-1}\right): 3084$ (N-H str), 1648 (C=O str), 1321 (asymm. $\mathrm{S}=\mathrm{O}$ str), 1152 (symm. $\mathrm{S}=\mathrm{O}$ str); ${ }^{1} \mathrm{H}$ NMR $\left(500 \mathrm{MHz}, \mathrm{CDCl}_{3}\right) \delta 7.95-7.93(\mathrm{~m}, 2 \mathrm{H}, \mathrm{Ar}-$ $\mathrm{H}+\mathrm{CH}=\mathrm{CH}), 7.88-7.86(\mathrm{~m}, 2 \mathrm{H}, \mathrm{Ar}-\mathrm{H}), 7.71(\mathrm{~d}, J 15.6 \mathrm{~Hz}, 1 \mathrm{H}, \mathrm{CH}=\mathrm{CH}), 7.61-7.56(\mathrm{~m}, 2 \mathrm{H}, \mathrm{Ar}-$ $\mathrm{H}), 7.50-7.44$ (m, 4H, Ar-H), $7.38-7.33$ (m, 2H, Ar-H), $7.22-7.19$ (m, 2H, Ar-H); ${ }^{13} \mathrm{C}$ NMR $\left(125 \mathrm{MHz}, \mathrm{CDCl}_{3}\right) \delta 188.37,143.07,140.93,136.63,134.12,133.47,130.39,130.21,129.27$, 127.84, 127.19, 126.79, 122.68, 119.29; Anal. calcd for $\mathrm{C}_{21} \mathrm{H}_{16} \mathrm{ClNO}_{3} \mathrm{~S}$ (397.87): C, 63.39; $\mathrm{H}$, 4.05 ; N, $3.52 \%$. Found: C, 63.22; H, 3.92; N, $3.60 \%$.

$\mathrm{N}$-(4-cinnamoylphenyl)-4-methylbenzenesulfonamide (5l). Yellow solid, yield 83\%, mp 162 $163^{\circ} \mathrm{C}$ (lit $\left.160-161^{\circ} \mathrm{C}\right)^{41}$, Rf 0.4 (30\% EtOAc:Hexane); IR ( $\left.v_{\max }, \mathrm{cm}^{-1}\right): 3219$ (N-H str), 3032 (Ar C-H str), 1666 (C=C str), 1336 (asymm. $\mathrm{S}=\mathrm{O}$ str), 1163 (symm. $\mathrm{S}=\mathrm{O}$ str); ${ }^{1} \mathrm{H}$ NMR (500 MHz, $\left.\mathrm{CDCl}_{3}\right) \delta$ 7.94-7.92 (m, 2H, Ar-H+CH=CH), $7.80-7.75(\mathrm{~m}, 3 \mathrm{H}, \mathrm{Ar}-\mathrm{H}), 7.62-7.61(\mathrm{~m}, 2 \mathrm{H}, \mathrm{Ar}-$ H), $7.47(\mathrm{~d}, J 15.6 \mathrm{~Hz}, 1 \mathrm{H}, \mathrm{CH}=\mathrm{CH}), 7.42-7.40(\mathrm{~m}, 3 \mathrm{H}, \mathrm{Ar}-\mathrm{H}), 7.27-7.21(\mathrm{~m}, 4 \mathrm{H}, \mathrm{Ar}-\mathrm{H}), 2.38$ $\left(\mathrm{s}, 3 \mathrm{H}, \mathrm{CH}_{3}\right) ;{ }^{13} \mathrm{C} \mathrm{NMR}\left(125 \mathrm{MHz}, \mathrm{CDCl}_{3}\right) \delta 188.90,144.82,144.44,140.99,135.85,134.79$, 134.21, 130.58, 130.15, 129.87, 128.94, 128.42, 127.26, 121.52, 119.11, 21.54; Anal. calcd for $\mathrm{C}_{22} \mathrm{H}_{19} \mathrm{NO}_{3} \mathrm{~S}$ (377.45): C, 70.01; H, 5.07; N, $3.71 \%$. Found: C, 70.21; H, 4.92; N, $3.61 \%$.

$\mathrm{N}$-(4-(3-(4-isopropylphenyl)acryloyl)phenyl)-4-methylbenzenesulfonamide $\quad \mathbf{( 5 m )}$ Yellow

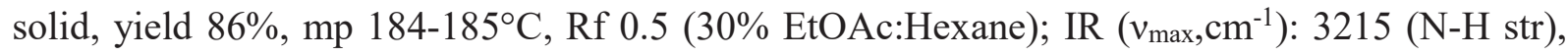
3049 (Ar C-H str), 1670 (C=O str), 1328 (asymm. S=O str), 1157 (symm. S=O str); ${ }^{1} \mathrm{H}$ NMR (500 $\left.\mathrm{MHz}, \mathrm{CDCl}_{3}\right) \delta 7.93-7.92(\mathrm{~m}, 2 \mathrm{H}, \mathrm{Ar}-\mathrm{H}+\mathrm{CH}=\mathrm{CH}), 7.79-7.74(\mathrm{~m}, 3 \mathrm{H}, \mathrm{Ar}-\mathrm{H}), 7.56(\mathrm{~d}, J 8.1$ $\mathrm{Hz}, 2 \mathrm{H}, \mathrm{Ar}-\mathrm{H}), 7.43$ (d, J 15.6 Hz, 1H, CH=CH), $7.28-7.25$ (m, 4H, Ar-H), $7.21-7.19$ (m, 2H, Ar-H), 2.94 (hept, J 7.2 Hz, 1H, CH(CH3) $\left.)_{2}\right), 2.38$ (s, 3H, CH $)_{3}, 1.27$ (d, J 6.9 Hz, 6H, CH(CH3) $)_{2}$; ${ }^{13} \mathrm{C}$ NMR $\left(125 \mathrm{MHz}, \mathrm{CDCl}_{3}\right) \delta 188.99,152.07,144.95,144.43,140.80,135.88,134.49,132.46$, $130.11,129.87,128.60,127.27,127.10,120.62,119.20,34.14,23.74,21.55$; Anal. calcd for $\mathrm{C}_{25} \mathrm{H}_{25} \mathrm{NO}_{3} \mathrm{~S}$ (419.53): C, 71.57; H, 6.01; N, 3.34 \%. Found: C, 71.68; H, 5.89; N, $3.49 \%$. 
4-methyl- $N$-(4-(3-(3,4,5-trimethoxyphenyl)acryloyl)phenyl)benzensulfonamide (5n). Yellow solid, yield 89\%, mp 195-197 C, Rf 0.4 (30\% EtOAc:Hexane); IR ( $\left.v_{\max }, \mathrm{cm}^{-1}\right): 3223$ (N-H str), 1651 (C=O str), 1402 (asymm. $\mathrm{S}=\mathrm{O}$ str), 1153 (symm. $\mathrm{S}=\mathrm{O}$ str); ${ }^{1} \mathrm{H}$ NMR $\left(500 \mathrm{MHz}, \mathrm{CDCl}_{3}\right) \delta 7.93-$ $7.91(\mathrm{~m}, 2 \mathrm{H}, \mathrm{Ar}-\mathrm{H}+\mathrm{CH}=\mathrm{CH}), 7.77-7.68(\mathrm{~m}, 3 \mathrm{H}, \mathrm{Ar}-\mathrm{H}), 7.34(\mathrm{~d}, J 15.5 \mathrm{~Hz}, 1 \mathrm{H}, \mathrm{CH}=\mathrm{CH}), 7.27-$ $7.20(\mathrm{~m}, 4 \mathrm{H}, \mathrm{Ar}-\mathrm{H}), 6.84(\mathrm{~s}, 2 \mathrm{H}, \mathrm{Ar}-\mathrm{H}), 3.91\left(\mathrm{~s}, 6 \mathrm{H}, \mathrm{OCH}_{3}\right), 3.90\left(\mathrm{~s}, 3 \mathrm{H}, \mathrm{OCH}_{3}\right), 2.38\left(\mathrm{~s}, 3 \mathrm{H}, \mathrm{CH}_{3}\right)$; ${ }^{13} \mathrm{C} \mathrm{NMR}\left(125 \mathrm{MHz}, \mathrm{CDCl}_{3}\right) \delta 188.92,153.47,145.02,144.42,140.99,135.91,134.22,130.25$, $130.12,129.86,127.24,120.85,119.08,105.73,60.98,56.23,21.53$; Anal. calcd for $\mathrm{C}_{25} \mathrm{H}_{25} \mathrm{NO}_{6} \mathrm{~S}$ (467.53): C, 64.23; H, 5.39; N, 3.00 \%. Found: C, 64.00; H, 5.65; N, $3.24 \%$.

4-methyl- $N$-(4-(3-(p-tolyl)acryloyl)phenyl)benzensulfonamide (5o). Yellow solid, yield 84\%, mp 208-209 ${ }^{\circ}$, Rf 0.58 (30\% EtOAc:Hexane); IR ( $\left.v_{\max }, \mathrm{cm}^{-1}\right): 3202$ (N-H str), 1600 (C=C str), 1333 (asymm. $\mathrm{S}=\mathrm{O}$ str), 1158 (symm. $\mathrm{S}=\mathrm{O}$ str); ${ }^{1} \mathrm{H}$ NMR $\left(500 \mathrm{MHz}, \mathrm{CDCl}_{3}\right) \delta 7.93-7.92$ (m, $2 \mathrm{H}, \mathrm{Ar}-\mathrm{H}+\mathrm{CH}=\mathrm{CH}), 7.78-7.74(\mathrm{~m}, 3 \mathrm{H}, \mathrm{Ar}-\mathrm{H}), 7.53-7.51(\mathrm{~m}, 2 \mathrm{H}, \mathrm{Ar}-\mathrm{H}), 7.43(\mathrm{~d}, J 15.6 \mathrm{~Hz}$, $1 \mathrm{H}, \mathrm{CH}=\mathrm{CH}), 7.27-7.19(\mathrm{~m}, 6 \mathrm{H}, \mathrm{Ar}-\mathrm{H}), 2.39\left(\mathrm{~s}, 3 \mathrm{H}, \mathrm{CH}_{3}\right), 2.38\left(\mathrm{~s}, 3 \mathrm{H}, \mathrm{CH}_{3}\right) ;{ }^{13} \mathrm{C} \mathrm{NMR}(125$ $\left.\mathrm{MHz}, \mathrm{CDCl}_{3}\right) \delta 188.99,144.93,144.42,141.16,140.85,135.87,134.42,132.07,130.10,129.87$, 129.70, 128.47, 127.26, 120.52, 119.17, 21.54, 21.52; Anal. calcd for $\mathrm{C}_{23} \mathrm{H}_{21} \mathrm{NO}_{3} \mathrm{~S}$ (391.48): $\mathrm{C}$, 70.57; H, 5.41; N, $3.58 \%$. Found: C, 70.36; H, 5.62; N, $3.71 \%$.

$\mathrm{N}$-(4-(3-(3-chlorophenyl)acryloyl)phenyl)-4-methylbenzenesulfonamide (5p). Yellow solid, yield 80\%, mp 213-214 ${ }^{\circ} \mathrm{C}$, Rf 0.6 (30\% EtOAc:Hexane); IR ( $\left.v_{\max }, \mathrm{cm}^{-1}\right): 3200$ (N-H str), 1658 $\left(\mathrm{C}=\mathrm{O}\right.$ str), 1333 (asymm. $\mathrm{S}=\mathrm{O}$ str), 1155 (symm. $\mathrm{S}=\mathrm{O}$ str); ${ }^{1} \mathrm{H}$ NMR $\left(500 \mathrm{MHz}, \mathrm{CDCl}_{3}\right) \delta 7.96-$ $7.91(\mathrm{~m}, 2 \mathrm{H}, \mathrm{Ar}-\mathrm{H}+\mathrm{CH}=\mathrm{CH}), 7.77-7.74(\mathrm{~m}, 2 \mathrm{H}, \mathrm{Ar}-\mathrm{H}), 7.71(\mathrm{~d}, J 15.7 \mathrm{~Hz}, 1 \mathrm{H}, \mathrm{CH}=\mathrm{CH}), 7.61$ - 7.60 (m, 1H, Ar-H), $7.49-7.43$ (m, 2H, Ar-H), 7.39-7.33 (m, 2H, Ar-H), 7.26 - 7.24 (m, 2H, Ar-H), 7.22- 7.19 (m,2H, ArH), 2.39 (s, 3H, $\left.\mathrm{CH}_{3}\right) ;{ }^{13} \mathrm{C} \mathrm{NMR}\left(125 \mathrm{MHz}, \mathrm{CDCl}_{3}\right) \delta 188.39,144.50$, $143.01,141.14,136.65,135.84,134.99,133.93,130.37,130.21,129.96,129.89,127.84,127.27$, 127.24, 126.78, 122.71, 119.09, 118.97, 21.55; Anal. calcd for $\mathrm{C}_{22} \mathrm{H}_{18} \mathrm{ClNO}_{3} \mathrm{~S}$ (411.90): C, 64.15; H, 4.40; N, $3.40 \%$. Found: C, 64.99; H, 4.42; N, $3.64 \%$.

$\mathrm{N}$-(4-(3-(4-chlorophenyl)acryloyl)phenyl)methanesulfonamide (5q). Yellow solid, yield 75\%, mp 159-161 ${ }^{\circ} \mathrm{C}$, Rf 0.6 (30\% EtOAc:Hexane); IR ( $\left.v_{\max }, \mathrm{cm}^{-1}\right): 3183$ (N-H str), 1651 (C=O str), 1340 (asymm. $\mathrm{S}=\mathrm{O}$ str), 1140 (symm. $\mathrm{S}=\mathrm{O}$ str); ${ }^{1} \mathrm{H}$ NMR $\left(500 \mathrm{MHz}, \mathrm{CDCl}_{3}\right) \delta 8.05$ (d, $J 8.2 \mathrm{~Hz}, 2 \mathrm{H}$, Ar-H), 7.77 (d, J $15.6 \mathrm{~Hz}, 1 \mathrm{H}, \mathrm{CH}=\mathrm{CH}), 7.58$ (d, J 8.1 Hz, 2H, Ar-H), 7.48 (d, J $15.6 \mathrm{~Hz}, 1 \mathrm{H}$, $\mathrm{CH}=\mathrm{CH}), 7.40(\mathrm{~d}, J 8.1 \mathrm{~Hz}, 2 \mathrm{H}, \mathrm{Ar}-\mathrm{H}), 7.30(\mathrm{~d}, J 8.4 \mathrm{~Hz}, 2 \mathrm{H}, \mathrm{Ar}-\mathrm{H}), 3.11\left(\mathrm{~s}, 3 \mathrm{H}, \mathrm{CH}_{3}\right) ;{ }^{13} \mathrm{C} \mathrm{NMR}$ $\left(125 \mathrm{MHz}, \mathrm{CDCl}_{3}\right) \delta 188.85,140.18,134.73,130.51,129.40,128.74,127.19,125.17,124.22$, 119.24, 40.20; Anal. calcd for $\mathrm{C}_{16} \mathrm{H}_{14} \mathrm{ClNO}_{3} \mathrm{~S}$ (335.80): C, 57.23; H, 4.20; N, 4.17 \%. Found: $\mathrm{C}$, 57.47 ; H, 4.34; N, $3.95 \%$.

N-(4-(3-(3,4,5-trimethoxyphenyl)acryloyl)phenyl)methanesulfonamide (5r). Yellow solid, yield 78\%, mp 170-172 ${ }^{\circ} \mathrm{C}$, Rf 0.4 (30\% EtOAc:Hexane); IR ( $\left.v_{\max }, \mathrm{cm}^{-1}\right): 3208$ (N-H str), 1643 $\left(\mathrm{C}=\mathrm{O}\right.$ str), 1318 (asymm. $\mathrm{S}=\mathrm{O}$ str), 1115 (symm. $\mathrm{S}=\mathrm{O}$ str); ${ }^{1} \mathrm{H}$ NMR $(500 \mathrm{MHz}$, Chloroform- $d$ ) $\delta$ $8.06-8.04$ (m, 2H. Ar-H), 7.77 (d, J 15.7 Hz, 1H, CH=CH), $7.60-7.56$ (m, 2H, Ar-H), 7.48 (d, $J 15.6 \mathrm{~Hz}, 1 \mathrm{H}, \mathrm{CH}=\mathrm{CH}), 7.32-7.28(\mathrm{~m}, 2 \mathrm{H}, \mathrm{Ar}-\mathrm{H}), 3.92\left(\mathrm{~s}, 6 \mathrm{H}, \mathrm{OCH}_{3}\right), 3.91\left(\mathrm{~s}, 3 \mathrm{H}, \mathrm{OCH}_{3}\right), 3.11$ $\left(\mathrm{s}, 3 \mathrm{H}, \mathrm{CH}_{3}\right) ;{ }^{13} \mathrm{C} \mathrm{NMR}\left(125 \mathrm{MHz}, \mathrm{CDCl}_{3}\right) \delta 188.99,151.36,134.53,130.36,129.19,128.92$, 
127.22, 125.12, 124.22, 119.26, 99.98, 61.36, 40.16, 30.75; Anal. calcd for $\mathrm{C}_{19} \mathrm{H}_{21} \mathrm{NO}_{6} \mathrm{~S}(391.43)$ : C, 58.30; H, 5.41; N, 3.58 \%. Found: C, 58.52; H, 5.67; N, $3.32 \%$.

General procedure for the synthesis of cyclohexenones (7a-7r). A round-bottom flask was charged with sulfonamide chalcones (5a-5r) $(1 \mathrm{mmol})$, ethylacetoacetate $(1 \mathrm{mmol})$ and dry $\mathrm{K}_{2} \mathrm{CO}_{3}$ $\left(2 \mathrm{mmol}\right.$ ) and the mixture was heated at $80^{\circ} \mathrm{C}$ for specified time (Table 1). The reaction progress was monitored by TLC. After completion of reaction, reaction mass is extracted from ethanol and the filtrate was evaporated to dryness. The residue was recrystallized from ethanol to obtain products $7 \mathbf{a}-\mathbf{7 r}$ with high yield (up to $95 \%$ ).

Ethyl 6-phenyl-2-oxo-4-((4-phenylsulfonamido)phenyl)-cyclohex-3-enecarboxylate (7a). Yellow solid, yield 91\%, 0.43g, mp 180-181 ${ }^{\circ} \mathrm{C}$ (from EtOH), Rf 0.54 (30\% EtOAc:Hexane); IR $\left(v_{\max }, \mathrm{cm}^{-1}\right): 3217(\mathrm{~N}-\mathrm{H}$ str), 1732 (ester $\mathrm{C}=\mathrm{O}$ str), 1655 (ketone $\mathrm{C}=\mathrm{O}$ str), 1372 (assym. $\mathrm{S}=\mathrm{O}$ str), 1080 (symm. $\mathrm{S}=\mathrm{O}$ str). ${ }^{1} \mathrm{H}$ NMR (400MHz, DMSO- $\left.d_{6}\right)$ : 7.67-7.65 (m, 2H, Ar-H), 7.38-7.20 (m, $10 \mathrm{H}, \mathrm{Ar}-\mathrm{H}), 6.77-6.75(\mathrm{~m}, 2 \mathrm{H}, \mathrm{Ar}-\mathrm{H}), 6.30\left(\mathrm{~s}, 1 \mathrm{H}, \mathrm{H}_{5}\right), 3.94-3.84\left(\mathrm{~m}, 3 \mathrm{H}, \mathrm{H}_{4}+\mathrm{OCH}_{2} \mathrm{CH}_{3}\right), 3.53-$ $3.45\left(\mathrm{~m}, 1 \mathrm{H}, \mathrm{H}_{3}\right), 2.90-2.95\left(\mathrm{~m}, 2 \mathrm{H}, \mathrm{H}_{1}+\mathrm{H}_{2}\right), 0.89$ (t, $\left.3 \mathrm{H}, J 7.2 \mathrm{~Hz}, \mathrm{OCH}_{2} \mathbf{C H}_{3}\right) ;{ }^{13} \mathrm{C} \mathrm{NMR}^{(100}$ MHz, DMSO-d $)$ : 193.24, 169.63, 154.95, 147.13, 141.95, 129.13, 128.36, 128.26, 127.86, $126.85,126.74,125.96,122.69,120.08,118.70,117.07,59.68,58.73,34.52,18.50,13.79$; MS (ESI): $m / z[\mathrm{M}+\mathrm{Na}]^{+}$calcd for $\mathrm{C}_{27} \mathrm{H}_{25} \mathrm{NNaO}_{5} \mathrm{~S}$ is 498.14 , found 498.22 ; Anal. calcd for $\mathrm{C}_{27} \mathrm{H}_{25} \mathrm{NO}_{5} \mathrm{~S}$ (475.14): C, 68.19; H, 5.30; N, $2.95 \%$. Found: C, 68.28; H, 5.36; N, $2.89 \%$.

Ethyl 6-(4-chlorophenyl)-2-oxo-4-((4-phenylsulfonamido)phenyl)-cyclohex-3-enecarboxyla te (7b). Yellow solid, yield $80 \%, 0.40 \mathrm{~g}, \mathrm{mp} 235-236{ }^{\circ} \mathrm{C}$ (from EtOH), Rf 0.44 (30\% EtOAc:Hexane); IR $\left(v_{\max }, \mathrm{cm}^{-1}\right): 3288$ (N-H str), 1732 (ester $\mathrm{C}=\mathrm{O}$ str), 1658 (ketone $\mathrm{C}=\mathrm{O}$ str), 1369 (assym. $\mathrm{S}=\mathrm{O}$ str), 1087 (symm. $\mathrm{S}=\mathrm{O}$ str); ${ }^{1} \mathrm{H}$ NMR (400MHz, DMSO-d $)$ ) 7.75-7.74 (m, 2H, Ar$\mathrm{H})$, 7.47-7.39 (m, 9H, Ar-H), 6.99-6.97 (m, 2H, Ar-H), 6.41 (s, 1H, H5), 4.00 (m, 2H, OCH $\mathrm{CH}_{3}$ ), $3.94\left(\mathrm{~m}, 2 \mathrm{H}, \mathrm{H}_{3} \mathrm{H}_{4}\right), 2.94\left(\mathrm{~m}, 2 \mathrm{H}, \mathrm{H}_{1}, \mathrm{H}_{2}\right), 0.92\left(\mathrm{t}, 3 \mathrm{H}, J 7.2 \mathrm{~Hz}, \mathrm{OCH}_{2} \mathbf{C H}_{3}\right) ;{ }^{13} \mathrm{C} \mathrm{NMR}(100 \mathrm{MHz}$, DMSO-d $d_{6}$ : $193.68,169.38,158.61,142.73,140.81,131.60,129.61,128.79,128.36,127.49$, $126.40,119.63,119.49,60.01,58.43,43.00,34.50,13.89 ; \mathrm{MS}(\mathrm{ESI}): \mathrm{m} / z[\mathrm{M}+\mathrm{Na}]^{+}$calcd for $\mathrm{C}_{27} \mathrm{H}_{24} \mathrm{ClNNaO}_{5} \mathrm{~S} 532.10$, observed 532.09; Anal. Calcd for $\mathrm{C}_{27} \mathrm{H}_{24} \mathrm{ClNO}_{5} \mathrm{~S}$ (509.10): C, 63.59; $\mathrm{H}$, $4.74 ;$ N, $2.75 \%$. Found: C, 63.43; H, 4.89; N, $2.84 \%$.

Ethyl 6-(4-fluorophenyl)-2-oxo-4-((4-phenylsulfonamido)phenyl)-cyclohex-3-enecarboxyla te (7c). Yellow solid, yield 88\%, 0.39g, mp 254-255 ${ }^{\circ} \mathrm{C}$ (from EtOH), Rf 0.46 (30\% EtOAc:Hexane); IR $\left(v_{\max }, \mathrm{cm}^{-1}\right): 3116(\mathrm{~N}-\mathrm{H}$ str), 1731 (ester $\mathrm{C}=\mathrm{O}$ str), 1655 (ketone $\mathrm{C}=\mathrm{O}$ str), 1372 (assym. S=O str), 1084 (symm. $\mathrm{S}=\mathrm{O}$ str); ${ }^{1} \mathrm{H}$ NMR (400MHz, DMSO- $\left.d_{6}\right)$ : 7.71-7.68 (m, 2H, ArH), 7.44-7.33 (m, 7H, Ar-H), 7.14-7.10 (m, 2H, Ar-H), 6.84-6.82 (m, 2H, Ar-H), $6.33\left(\mathrm{~s}, 1 \mathrm{H}, \mathrm{H}_{5}\right)$, $4.102\left(\mathrm{~m}, 1 \mathrm{H}, \mathrm{H}_{4}\right), 3.95-3.85\left(\mathrm{~m}, 2 \mathrm{H}, \mathrm{OCH}_{2} \mathrm{CH}_{3}\right), 3.56-3.49\left(\mathrm{~m}, 1 \mathrm{H}, \mathrm{H}_{3}\right), 2.94-2.91\left(\mathrm{~m}, 2 \mathrm{H}, \mathrm{H}_{1}\right.$, $\mathrm{H}_{2}$ ), 0.92 (t, $\left.3 \mathrm{H}, J 7.2 \mathrm{~Hz}, \mathrm{OCH}_{2} \mathbf{C H}_{3}\right) ;{ }^{13} \mathrm{C} \mathrm{NMR}$ (100 MHz, DMSO-d $)$ ): 193.30, 169.53, 162.32, 159.09, 152.08, 145.60, 139.74, 138.60, 129.50, 128.92, 128.03, 127.14, 126.97, 126.04, 117.97, $115.09,114.79,59.80,58.83,43.09,34.54,13.81 ; \mathrm{MS}(\mathrm{ESI}): \mathrm{m} / \mathrm{z}[\mathrm{M}+\mathrm{Na}]^{+}$calc. for 
$\mathrm{C}_{27} \mathrm{H}_{24} \mathrm{FNNaO}_{5} \mathrm{~S}$ is 516.13, found 516.38; Anal. calcd for $\mathrm{C}_{27} \mathrm{H}_{24} \mathrm{FNO}_{5} \mathrm{~S}$ (493.13): $\mathrm{C}, 65.71 ; \mathrm{H}$, $4.90 ; \mathrm{N}, 2.84 \%$. Found: C, 65.67; H, 5.01; N, $2.90 \%$.

Ethyl 6-(4-bromophenyl)-2-oxo-4-((4-phenylsulfonamido)phenyl)-cyclohex-3-enecarboxyla te (7d). Yellow solid, yield 80\%, 0.44g, mp 270-272 ${ }^{\circ} \mathrm{C}$ (from EtOH), Rf 0.46 (30\% EtOAc:Hexane); IR $\left(v_{\max }, \mathrm{cm}^{-1}\right): 3115$ (N-H str), 1732 (ester $\mathrm{C}=\mathrm{O}$ str), 1656 (ketone $\mathrm{C}=\mathrm{O}$ str), 1369 (assym. $\mathrm{S}=\mathrm{O}$ str), 1084 (symm. $\mathrm{S}=\mathrm{O}$ str); ${ }^{1} \mathrm{H}$ NMR (400MHz, DMSO- $\left.d_{6}\right)$ : 7.68-6.74 (m, 13H, Ar$\mathrm{H}), 6.30\left(\mathrm{~s}, 1 \mathrm{H}, \mathrm{H}_{5}\right), 3.95-3.86\left(\mathrm{~m}, 3 \mathrm{H}, \mathrm{H}_{4}+\mathrm{OCH}_{2} \mathrm{CH}_{3}\right), 3.54-3.48\left(\mathrm{~m}, 1 \mathrm{H}, \mathrm{H}_{3}\right), 2.88-2.84(\mathrm{~m}, 2 \mathrm{H}$, $\mathrm{H}_{1}+\mathrm{H}_{2}$ ), 0.93 (t, 3H, J $\left.6.8 \mathrm{~Hz}, \mathrm{OCH}_{2} \mathbf{C H}_{3}\right) ;{ }^{13} \mathrm{C}$ NMR (100 MHz, DMSO-d 6 ): 192.99, 169.54, 159.21, 154.03, 146.98, 141.40, 131.17, 129.22, 127.90, 126.01, 122.74, 120.10, 119.95, 117.09, 59.84, 58.46, 43.23, 34.22, 13.83; MS (ESI): $\mathrm{m} / z$ [M+Na] $]^{+}$calcd for $\mathrm{C}_{27} \mathrm{H}_{24} \mathrm{BrNNaO}_{5} \mathrm{~S}$ is 576.05, found 576.16; Anal. Calcd for $\mathrm{C}_{27} \mathrm{H}_{24} \mathrm{BrNO}_{5} \mathrm{~S}$ (553.05): C, 58.49; H, 4.36; N, 2.53 \%. Found: $\mathrm{C}$, 58.32; H, 4.59; N, $2.33 \%$.

Ethyl 6-(4-isopropylphenyl)-2-oxo-4-((4-phenylsulfonamido)phenyl)-cyclohex-3-enecarboxy -late (7e). Yellow solid, yield 95\%, 0.49g, mp 199-200 ${ }^{\circ} \mathrm{C}$ (from $\left.\mathrm{EtOH}\right), \mathrm{Rf} 0.46$ (30\% EtOAc:Hexane); IR $\left(v_{\max }, \mathrm{cm}^{-1}\right): 3015$ (N-H str), 1725 (ester $\mathrm{C}=\mathrm{O}$ str), 1634 (ketone $\mathrm{C}=\mathrm{O}$ str), 1372 (assym. $\mathrm{S}=\mathrm{O}$ str), 1082 (symm. $\mathrm{S}=\mathrm{O}$ str); ${ }^{1} \mathrm{H}$ NMR (400MHz, DMSO- $\left.d_{6}\right): 7.68-7.65$ (m, 2H, ArH), 7.34-7.26 (m, 7H, Ar-H), 7.18-7.15 (m, 2H, Ar-H), 6.76-6.74 (m, 2H, Ar-H), 6.29 (s, 1H, $\left.\mathrm{H}_{5}\right)$, 3.92-3.83 (m, 3H, $\left.\mathrm{H}_{4}+\mathrm{OCH}_{2} \mathrm{CH}_{3}\right), 3.71-3.49\left(\mathrm{~m}, 1 \mathrm{H}, \mathrm{H}_{3}\right), 2.97-2.81\left(\mathrm{~m}, 3 \mathrm{H}, \mathbf{C H}\left(\mathrm{CH}_{3}\right)_{2}+\mathrm{H}_{1}+\mathrm{H}_{2}\right)$, 1.19-1.16 (m, 6H, CH(CH$\left.)_{2}\right), 0.87$ (t, $\left.3 \mathrm{H}, J 7.2 \mathrm{~Hz}, \mathrm{OCH}_{2} \mathbf{C H}_{3}\right) ;{ }^{13} \mathrm{C}$ NMR (100 MHz, DMSO$\left.d_{6}\right)$ : 193.83, 169.71, 159.39, 158.75, 154.38, 147.87, 141.50, 139.29, 129.16, 127.43, 126.74, 123.53, 120.09, 59.64, 58.84, 43.51, 40.12, 34.67, 33.00, 23.84, 23.84, 13.73; MS (ESI): $\mathrm{m} / \mathrm{z}$ $[\mathrm{M}+\mathrm{Na}]^{+}$calcd for $\mathrm{C}_{30} \mathrm{H}_{31} \mathrm{NNaO}_{5} \mathrm{~S}$ is 540.18 , found 540.20; Anal. calcd for $\mathrm{C}_{30} \mathrm{H}_{31} \mathrm{NO}_{5} \mathrm{~S}(527.18)$ : C, 69.61; H, 6.04; N, $2.71 \%$. Found: C, 69.43; H, 6.23; N, $2.45 \%$.

Ethyl 6-(2-furyl)-2-oxo-4-((4-phenylsulfonamido)phenyl)-cyclohex-3-enecarboxylate (7f). Yellow solid, yield 72\%, 0.33g, mp 196-198 ${ }^{\circ} \mathrm{C}$ (from EtOH), Rf 0.40 (30\% EtOAc:Hexane); IR $\left(v_{\max }, \mathrm{cm}^{-1}\right): 3011(\mathrm{~N}-\mathrm{H}$ str), 1716 (ester $\mathrm{C}=\mathrm{O}$ str), 1625 (ketone $\mathrm{C}=\mathrm{O}$ str), 1372 (assym. $\mathrm{S}=\mathrm{O}$ str), 1080 (symm. $\mathrm{S}=\mathrm{O}$ str); ${ }^{1} \mathrm{H}$ NMR (400MHz, DMSO- $\left.d_{6}\right)$ : 7.69-7.68 (m, 3H, Ar-H), 7.35-7.33 (m, $6 \mathrm{H}, \mathrm{ArH}+$ furyl H), 6.80-6.77 (m, 2H, Ar-H), 6.39-6.30 (m, 2H, Furyl H+ $\left.\mathrm{H}_{5}\right), 6.23-6.22(\mathrm{~m}, 1 \mathrm{H}$, Furyl H), 4.05-4.03 (m, 2H, $\left.\mathrm{H}_{4}+\mathrm{OCH}_{2} \mathrm{CH}_{3}\right), 3.68\left(\mathrm{~m}, 1 \mathrm{H}, \mathrm{H}_{3}\right), 3.12\left(\mathrm{~m}, 1 \mathrm{H}, \mathrm{H}_{2}\right), 2.89(\mathrm{~m}, 1 \mathrm{H}$, $\left.\mathrm{H}_{1}\right), 1.086$ (t, $\left.3 \mathrm{H}, J 6.8 \mathrm{~Hz}, \mathrm{OCH}_{2} \mathbf{C H}_{3}\right) ;{ }^{13} \mathrm{C}$ NMR (100 MHz, DMSO-d $)$ ): 192.35, 169.67, 158.77, 155.67, 155.02, 147.64, 141.93, 129.65,129.27, 127.98, 126.65, 122.64, 120.13, 117.17, 110.37, $105.70,60.20,57.11,38.88,13.98$; MS (ESI): $m / z$ [M+Na $]^{+}$calcd for $\mathrm{C}_{25} \mathrm{H}_{23} \mathrm{NNaO}_{6} \mathrm{~S}$ is 488.11 found 488.11; Anal. calcd for $\mathrm{C}_{25} \mathrm{H}_{23} \mathrm{NO}_{6} \mathrm{~S}$ (465.11): C, 64.50; H, 4.98; N, $3.01 \%$. Found: $\mathrm{C}$, $64.80 ; \mathrm{H}, 4.86 ; \mathrm{N}, 3.14 \%$.

Ethyl 6-(3,4,5-trimethoxyphenyl)-2-oxo-4-((4-phenylsulfonamido)phenyl)-cyclohex-3-ene carboxylate (7g). Yellow solid, yield $80 \%, 0.45 \mathrm{~g}, \mathrm{mp} 241-243{ }^{\circ} \mathrm{C}$ (from EtOH), Rf 0.32 (30\% EtOAc:Hexane); IR $\left(v_{\max }, \mathrm{cm}^{-1}\right): 3107$ (N-H str), 1720 (ester $\mathrm{C}=\mathrm{O}$ str), 1630 (ketone $\mathrm{C}=\mathrm{O}$ str), 1337 (assym. S=O str), 1129 (symm. S=O str); ${ }^{1} \mathrm{H}$ NMR (400MHz, DMSO-d $)$ : 10.64 (s, 1H, NH), 7.81$6.71(\mathrm{~m}, 11 \mathrm{H}, \mathrm{Ar}-\mathrm{H}), 6.38\left(\mathrm{~s}, 1 \mathrm{H}, \mathrm{H}_{5}\right), 3.76\left(\mathrm{~s}, 6 \mathrm{H}, \mathrm{OCH}_{3}\right), 3.70\left(\mathrm{~s}, 3 \mathrm{H}, \mathrm{OCH}_{3}\right), 3.30-3.35(\mathrm{~m}, 3 \mathrm{H}$, $\left.\mathrm{H}_{4}+\mathrm{OCH}_{2} \mathrm{CH}_{3}\right), 2.92\left(\mathrm{~m}, 1 \mathrm{H}, \mathrm{H}_{3}\right), 2.82\left(\mathrm{~m}, 1 \mathrm{H}, \mathrm{H}_{1}\right), 2.50\left(\mathrm{~m}, 1 \mathrm{H}, \mathrm{H}_{2}\right), 0.93(\mathrm{t}, 3 \mathrm{H}, J 7.64 \mathrm{~Hz}$, 
$\left.\mathrm{OCH}_{2} \mathbf{C H}_{3}\right) ;{ }^{13} \mathrm{C}$ NMR (100 MHz, DMSO- $\left.d_{6}\right): 198.30,157.59,152.81,139.63,139.42,136.09$, 132.81, 129.37, 127.59, 126.61, 122.98, 119.03, 104.41, 60.91, 59.92, 57.59, 55.84, 43.50, 35.00, 25.84; MS (ESI): $m / z$ [M+Na] $]^{+}$calc. for $\mathrm{C}_{30} \mathrm{H}_{31} \mathrm{NNaO}_{8} \mathrm{~S}$ is 588.17 , found 588.15; Anal. calcd for $\mathrm{C}_{30} \mathrm{H}_{31} \mathrm{NO}_{8} \mathrm{~S}$ (565.17): C, 63.70; H, 5.52; N, $2.48 \%$. Found: C, 63.55; H, 5.78; N, $2.31 \%$.

Ethyl 6-(2-chlorophenyl)-2-oxo-4-((4-phenylsulfonamido)phenyl)-cyclohex-3-enecarboxyla te (7h). Yellow solid, yield 81\%, 0.41g, mp 210-211 ${ }^{\circ} \mathrm{C}$ (from EtOH), Rf 0.44 (30\% EtOAc:Hexane); IR $\left(v_{\max }, \mathrm{cm}^{-1}\right): 3402(\mathrm{~N}-\mathrm{H}$ str), 1720 (ester $\mathrm{C}=\mathrm{O}$ str), 1636 (ketone $\mathrm{C}=\mathrm{O}$ str), 1370 (assym. $\mathrm{S}=\mathrm{O}$ str), 1080 (symm. $\mathrm{S}=\mathrm{O}$ str); ${ }^{1} \mathrm{H}$ NMR (400MHz, DMSO-d $)$ : 7.68-7.66 (m, 2H , ArH), 7.62-7.60 (m, 1H , Ar-H), 7.44-7.41 (m, 1H , Ar-H), 7.35-7.25(m, 7H , Ar-H), 6.77-6.75 (m, $2 \mathrm{H}, \mathrm{Ar}-\mathrm{H}), 6.33\left(\mathrm{~s}, 1 \mathrm{H}, \mathrm{H}_{5}\right), 4.18\left(\mathrm{~m}, 1 \mathrm{H}, \mathrm{H}_{4}\right), 3.89-3.86\left(\mathrm{~m}, 3 \mathrm{H}, \mathrm{H}_{3}+\mathrm{OCH}_{2} \mathrm{CH}_{3}\right), 2.93-2.48(\mathrm{~m}$, $\left.2 \mathrm{H}, \mathrm{H}_{1}+\mathrm{H}_{2}\right), 0.89$ (t, $\left.3 \mathrm{H}, J 8.0 \mathrm{~Hz}, \mathrm{OCH}_{2} \mathbf{C H}_{3}\right) ;{ }^{13} \mathrm{C}$ NMR (100 MHz, DMSO-d $)$ ): 197.90, 169.30, $158.82,155.07,147.05,138.89,132.81,129.45,129.20,128.52,127.90,127.45,126,76,125.98$, $122.98,122.14,116.98,59.87,57.45,42.40,33.32,13.71$; MS (ESI): $m / z[\mathrm{M}+\mathrm{H}]^{+}$calcd for $\mathrm{C}_{27} \mathrm{H}_{25} \mathrm{ClNO}_{5} \mathrm{~S}$ is 510.11, found 510.31; Anal. calcd for $\mathrm{C}_{27} \mathrm{H}_{24} \mathrm{ClNO}_{5} \mathrm{~S}$ (509.11): C, 63.59; $\mathrm{H}$, $4.74 ; \mathrm{N}, 2.75 \%$. Found: C, 63.43; H, 4.56; N, $2.87 \%$.

Ethyl 6-(2-bromophenyl)-2-oxo-4-((4-phenylsulfonamido)phenyl)-cyclohex-3-ene carboxyla -te (7i). Yellow solid, yield $80 \%, 0.44 \mathrm{~g}, \mathrm{mp} 224-226{ }^{\circ} \mathrm{C}$ (from $\left.\mathrm{EtOH}\right), \mathrm{Rf} 0.48$ (30\% EtOAc:Hexane); IR ( $\left.v_{\max }, \mathrm{cm}^{-1}\right): 3056$ (N-H str), 1720 (ester $\mathrm{C}=\mathrm{O}$ str), 1636 (ketone $\mathrm{C}=\mathrm{O}$ str), 1370 (assym. S=O str), 1080 (symm. $\mathrm{S}=\mathrm{O}$ str); ${ }^{1} \mathrm{H}$ NMR (400MHz, DMSO- $\left.d_{6}\right)$ : 7.68-7.65 (m, 3H, Ar$\mathrm{H})$, 7.62-7.54 (m, 3H, Ar-H), 7.40-7.16 (m, 3H, Ar-H), 6.33 (d, 1H, $\left.\mathrm{H}_{5}\right), 4.20-4.17\left(\mathrm{~m}, 1 \mathrm{H}, \mathrm{H}_{4}\right)$, 4.02-3.86 (m, 3H, $\left.\mathrm{H}_{3}+\mathrm{OCH}_{2} \mathrm{CH}_{3}\right), 2.98-2.65\left(\mathrm{~m}, 2 \mathrm{H}, \mathrm{H}_{1}+\mathrm{H}_{2}\right), 0.90\left(\mathrm{t}, 3 \mathrm{H}, J 6.8 \mathrm{~Hz}, \mathrm{OCH}_{2} \mathbf{C H}_{3}\right)$ ${ }^{13} \mathrm{C}$ NMR (100 MHz, DMSO- $d_{6}$ ): 197.10, 169.29, 158.79, 155.08, 147.19, 147.06, 129.21, 128.20, 127.91, 126.99, 126.77, 126.01, 120.15, 116.99, 59.88, 57.54, 42.42, 33.47, 33.04, 13.74; MS (ESI): $m / z[\mathrm{M}+\mathrm{H}]^{+}$calcd for $\mathrm{C}_{27} \mathrm{H}_{25} \mathrm{BrNO}_{5} \mathrm{~S}$ is 554.06, found 554.10; Anal. calcd for $\mathrm{C}_{27} \mathrm{H}_{24} \mathrm{BrNO}_{5} \mathrm{~S}$ (553.06): C, 58.49; H, 4.36; N, 2.53 \%. Found: C, 58.55; H, 4.18; N, $2.34 \%$.

Ethyl 6-(4-methylphenyl)-2-oxo-4-((4-phenylsulfonamido)phenyl)-cyclohex-3-enecarboxyla -te (7j). Yellow solid, yield 89\%, 0.44g, mp 193-194 ${ }^{\circ} \mathrm{C}$ (from EtOH), Rf 0.48 (30\% EtOAc:Hexane); IR $\left(v_{\max }, \mathrm{cm}^{-1}\right): 3122(\mathrm{~N}-\mathrm{H}$ str), 1733 (ester $\mathrm{C}=\mathrm{O}$ str), 1658 (ketone $\mathrm{C}=\mathrm{O}$ str), 1371 (assym. S=O str), 1080 (symm. S=O str); ${ }^{1} \mathrm{H}$ NMR (400MHz, DMSO- $\left.d_{6}\right): 7.71-7.69$ (m, 2H, ArH), 7.34-7.24 (m, 7H, Ar-H), 7.12-7.11 (m, 2H, Ar-H), 6.79-6.77 (m, 2H, Ar-H), $6.32\left(\mathrm{~s}, 1 \mathrm{H}, \mathrm{H}_{5}\right)$, 3.92-3.87 (m, 3H, H4 $\left.+\mathrm{OCH}_{2} \mathrm{CH}_{3}\right), 3.46-3.43\left(\mathrm{~m}, 1 \mathrm{H}, \mathrm{H}_{3}\right), 2.91-2.85\left(\mathrm{~m}, 2 \mathrm{H}, \mathrm{H}_{1}+\mathrm{H}_{2}\right), 2.26(\mathrm{~s}, 3 \mathrm{H}$, $\mathrm{CH}_{3}$ ), 0.93 (t, $3 \mathrm{H}, J 7.64 \mathrm{~Hz}, \mathrm{OCH}_{2} \mathbf{C H}_{3}$ ); ${ }^{13} \mathrm{C}$ NMR (100 MHz, DMSO- $d_{6}$ ): 193.42, 169.68, $159.36,154.80,147.00,138.97,135.90,129.23,128.85,127.92,127.39,126.02,117.18,59.73$, 58.83, 43.46, 39.28, 20.63, 13.87; MS (ESI): $m / z$ [M+Na $]^{+}$calcd for $\mathrm{C}_{28} \mathrm{H}_{27} \mathrm{NNaO}_{5} \mathrm{~S}$ is 512.15, observed 512.14; Anal. Calcd for $\mathrm{C}_{28} \mathrm{H}_{27} \mathrm{NO}_{5} \mathrm{~S}$ (489.15): C, 68.69; H, 5.56; N, $2.86 \%$. Found: $\mathrm{C}$, 68.74; H, 5.36; N, $2.90 \%$.

Ethyl 6-(3-chlorophenyl)-2-oxo-4-((4-phenylsulfonamido)phenyl)-cyclohex-3-enecarboxyla te (7k). Yellow solid, yield 90\%, 0.46g, mp 236-237 ${ }^{\circ} \mathrm{C}$ (from EtOH), Rf 0.46 (30\% EtOAc:Hexane); IR ( $\left.v_{\max }, \mathrm{cm}^{-1}\right): 3131$ (N-H str), 1736 (ester C=O str), 1650 (ketone $\mathrm{C}=\mathrm{O}$ str), 1372 (assym. $\mathrm{S}=\mathrm{O}$ str), 1085 (symm. $\mathrm{S}=\mathrm{O}$ str); ${ }^{1} \mathrm{H}$ NMR (400MHz, DMSO-d $)$ ): 7.71-7.69 (m, 2H, Ar- 
H), 7.51 (s, 1H, Ar-H), 7.36-7.29 (m, 9H, Ar-H), 6.80-6.78 (m, 1H, Ar-H), $6.33\left(\mathrm{~s}, 1 \mathrm{H}, \mathrm{H}_{5}\right), 4.02-$ $3.98\left(\mathrm{~m}, 1 \mathrm{H}, \mathrm{H}_{4}\right), 3.92-3.90\left(\mathrm{~m}, 2 \mathrm{H}, \mathrm{OCH}_{2} \mathrm{CH}_{3}\right), 3.58-3.54\left(\mathrm{~m}, 1 \mathrm{H}, \mathrm{H}_{3}\right), 2.98-2.90\left(\mathrm{~m}, 2 \mathrm{H}, \mathrm{H}_{1}+\mathrm{H}_{2}\right)$, 0.92 (t, 3H, J $6.48 \mathrm{~Hz}, \mathrm{OCH}_{2} \mathbf{C H}_{3}$ ); ${ }^{13} \mathrm{C}$ NMR (100 MHz, DMSO-d $)$ ): 193.00, 169.56, 159.26, $154.84,146.95,144.49$, 130.19, 129.27, 127.62, 126.92, 126.44, 120.12, 117.06, 59.84, 58.39, 43.50, 34.00, 13.85; MS (ESI): $\mathrm{m} / z$ [M+Na] ${ }^{+}$calcd for $\mathrm{C}_{27} \mathrm{H}_{24} \mathrm{ClNNaO}_{5} \mathrm{~S}$ are 532.10, found 532.10; Anal. calcd for $\mathrm{C}_{27} \mathrm{H}_{24} \mathrm{ClNO}_{5} \mathrm{~S}$ (509.01): C, 63.59; H, 4.74; N, 2.75 \%. Found: C, 63.48; H, 4.44; $\mathrm{N}, 2.50 \%$.

Ethyl 6-phenyl-2-oxo-4-((4-(4-methylphenylsulfonamido)phenyl))-cyclohex-3-enecarboxyl ate (7l). Yellow solid, yield $90 \%, 0.44 \mathrm{~g}, \mathrm{mp} \mathrm{180-181}{ }^{\circ} \mathrm{C}$ (From EtOH), Rf 0.60 (30\% EtOAc:Hexane); IR $\left(v_{\max }, \mathrm{cm}^{-1}\right)$ : 3235 (N-H str), 1724 (ester $\mathrm{C}=\mathrm{O}$ str), 1637 (ketone $\mathrm{C}=\mathrm{O}$ str), 1372 (assym. S=O str), 1079 (symm. S=O str); ${ }^{1} \mathrm{H}$ NMR (400MHz, DMSO- $\left.d_{6}\right)$ : 7.55-7.53 (m, 2H, ArH), 7.38-7.36 (m, 2H, Ar-H), 7.31-7.27 (m, 4H, Ar-H), 7.23-7.19 (m, 1H, Ar-H), 7.12-7.10 (m, 2H, Ar-H), 6.74-6.72 (m, 2H, Ar-H), 6.30 (s, $\left.1 \mathrm{H}, \mathrm{H}_{5}\right), 3.93-3.84$ (m, 3H, $\left.\mathrm{H}_{4}+\mathrm{OCH}_{2} \mathrm{CH}_{3}\right), 3.52-3.45$ (m, 1H, H3), 2.99-2.83 (m, 2H, $\left.\mathrm{H}_{1}+\mathrm{H}_{2}\right), 2.25\left(\mathrm{~s}, 3 \mathrm{H}, \mathrm{CH}_{3}\right), 0.89$ (t, 3H, J 7.2 Hz, $\left.\mathrm{OCH}_{2} \mathbf{C H}_{3}\right) ;{ }^{13} \mathrm{C}$ NMR (100 MHz, DMSO-d $)$ : 193.23. 169.64, 159.35, 155.05, 144.26, 141.95, 138.68, 128.33, $128.27,127.55,126.97,122.55,120.04,116.98,59.67,58.73,43.85,34.52,20.72,13.79$; MS (ESI): $m / z[\mathrm{M}+\mathrm{Na}]^{+}$calcd for $\mathrm{C}_{28} \mathrm{H}_{27} \mathrm{NNaO}_{5} \mathrm{~S}$ is 512.15 , found 512.34; Anal. calcd for $\mathrm{C}_{28} \mathrm{H}_{27} \mathrm{NO}_{5} \mathrm{~S}$ (489.15): C, 63.59; H, 4.74; N, $2.75 \%$. Found: C, 63.48; H, 4.44; N, $2.50 \%$.

Ethyl 6-(4-isopropylphenyl)-2-oxo-4-((4-(4-methylphenylsulfonamido)phenyl))-cyclohex-3enecarboxylate (7m). Yellow solid, yield $95 \%, 0.5 \mathrm{~g}, \mathrm{mp} 211-212{ }^{\circ} \mathrm{C}$ (from EtOH), Rf $0.35(30 \%$ EtOAc:Hexane); IR $\left(v_{\max }, \mathrm{cm}^{-1}\right): 3171(\mathrm{~N}-\mathrm{H}$ str), 1727 (ester $\mathrm{C}=\mathrm{O}$ str), 1635 (ketone $\mathrm{C}=\mathrm{O}$ str), 1351 (assym. $\mathrm{S}=\mathrm{O}$ str), 1083 (symm. $\mathrm{S}=\mathrm{O}$ str) $\mathrm{cm}^{-1} ;{ }^{1} \mathrm{H}$ NMR (400MHz, DMSO-d $)$ ) 7.66-7.64 (m, 2H, Ar-H), 7.52-7.50 (m, 2H, Ar-H), 7.29-7.27 (m, 4H, Ar-H), 7.18-7.16 (m, 2H, Ar-H), 7.04-7.02 (m, 2H, Ar-H), $6.42\left(\mathrm{~s}, 1 \mathrm{H}, \mathrm{H}_{5}\right), 3.99-3.96\left(\mathrm{~m}, 1 \mathrm{H}, \mathrm{H}_{4}\right), 3.91-3.89\left(\mathrm{~m}, 2 \mathrm{H}, \mathrm{OCH}_{2} \mathrm{CH}_{3}\right), 3.52-3.49(\mathrm{~m}$, $\left.1 \mathrm{H}, \mathrm{H}_{3}\right), 2.93-2.91\left(\mathrm{~m}, 2 \mathrm{H}, \mathrm{H}_{1}+\mathrm{H}_{2}\right), 2.84-2.82\left(\mathrm{~m}, 1 \mathrm{H}, \mathbf{C H}\left(\mathrm{CH}_{3}\right)_{2}\right), 2.30\left(\mathrm{~s}, 3 \mathrm{H}, \mathrm{CH}_{3}\right), 1.16(\mathrm{~d}, 6 \mathrm{H}$, $\left.J 6.84 \mathrm{~Hz}, \mathrm{CH}\left(\mathbf{C H}_{3}\right)_{2}\right), 0.87$ (t, $\left.3 \mathrm{H}, J 7.24 \mathrm{~Hz}, \mathrm{OCH}_{2} \mathbf{C H}_{3}\right) ;{ }^{13} \mathrm{C}$ NMR (100 MHz, DMSO- $\left.d_{6}\right)$ : 193.94, 169.44, 158.49, 147.17, 142.17, 138.99, 138.63, 129.38, 127.45, 126.18, 120.32, 119.12, 59.78, 58.78, 43.50, 34.00, 33.05, 23.84, 20.86, 13.73; MS (ESI): $\mathrm{m} / z[\mathrm{M}+\mathrm{Na}]^{+}$calcd for $\mathrm{C}_{31} \mathrm{H}_{33} \mathrm{NNaO}_{5} \mathrm{~S} 554.20$, observed 554.18; Anal. calcd for $\mathrm{C}_{31} \mathrm{H}_{33} \mathrm{NO}_{5} \mathrm{~S}$ (531.20): C, 70.03; H, 6.26; N, $2.63 \%$. Found: C, 70.33; H, 6.06; N, $2.40 \%$.

Ethyl 6-(3,4,5-trimethoxyphenyl)-2-oxo-4-((4-(4-methylphenylsulfonamido)phenyl))-cyclo hex-3-enecarboxylate (7n). Yellow solid, yield 93\%, 0.54g, mp 256-257 ${ }^{\circ} \mathrm{C}$ (from EtOH), Rf 0.20 (30\% EtOAc:Hexane); IR ( $\left.v_{\max }, \mathrm{cm}^{-1}\right): 3212$ (N-H str), 1729 (ester $\mathrm{C}=\mathrm{O}$ str), 1638 (ketone $\mathrm{C}=\mathrm{O}$ str), 1327 (assym. $\mathrm{S}=\mathrm{O}$ str), 1125 (symm. $\mathrm{S}=\mathrm{O}$ str) $\mathrm{cm}^{-1} ;{ }^{1} \mathrm{H}$ NMR (400MHz, DMSO-d $)$ ): 10.65 (s, 1H, NH), 7.69-7.62 (m, 4H, Ar-H), 7.35-7.33 (m, 2H, Ar-H), 7.16-7.14 (m, 2H, Ar-H), 6.72 (s, $2 \mathrm{H}, \mathrm{Ar}-\mathrm{H}), 6.47\left(\mathrm{~s}, 1 \mathrm{H}, \mathrm{H}_{5}\right), 4.12-4.09\left(\mathrm{~m}, 1 \mathrm{H}, \mathrm{H}_{4}\right), 3.96-3.94\left(\mathrm{~m}, 2 \mathrm{H}, \mathrm{OCH}_{2} \mathrm{CH}_{3}\right), 3.74(\mathrm{~s}, 6 \mathrm{H}$, $\left.\mathrm{OCH}_{3}\right), 3.62\left(\mathrm{~s}, 3 \mathrm{H}, \mathrm{OCH}_{3}\right), 3.56-3.52\left(\mathrm{~m}, 1 \mathrm{H}, \mathrm{H}_{3}\right), 2.96-2.91\left(\mathrm{~m}, 2 \mathrm{H}, \mathrm{H}_{1}+\mathrm{H}_{2}\right), 2.32\left(\mathrm{~s}, 3 \mathrm{H}, \mathrm{CH}_{3}\right)$, 0.96 (t, 3H, $\left.J 6.88 \mathrm{~Hz}, \mathrm{OCH}_{2} \mathbf{C H}_{3}\right) ;{ }^{13} \mathrm{C}$ NMR (100 MHz, DMSO-d $)$ ): 194.23, 169.44, 158.81, $152.71,143.55,140.14,137.35,136.51,136.30,131.89,131.89,129.82$, , 127.77, 126.70, 121.59, $118.80,104.90,104.40,60.44,59.94,58.55,55.86,43.50,35.00,20.95,13.93$; MS (ESI): $\mathrm{m} / z$ 
$[\mathrm{M}+\mathrm{Na}]^{+}$calcd for $\mathrm{C}_{31} \mathrm{H}_{33} \mathrm{NNaO}_{8} \mathrm{~S}$ 602.18, observed 602.17; Anal. calcd for $\mathrm{C}_{31} \mathrm{H}_{33} \mathrm{NO}_{8} \mathrm{~S}$ (579.18): C, 64.23; H, 5.74; N, $2.42 \%$. Found: C, 64.43; H, 5.87; N, 2.54; \%.

Ethyl 6-(4-methylphenyl)-2-oxo-4-((4-(4-methylphenylsulfonamido)phenyl))-cyclohex-3-ene carboxylate (7o). Yellow solid, yield 80\%, 0.4g, mp 201-202 ${ }^{\circ} \mathrm{C}$ (from EtOH), Rf 0.37 (30\% EtOAc:Hexane); IR ( $\left.v_{\max }, \mathrm{cm}^{-1}\right): 3234$ (N-H str), 1738 (ester $\mathrm{C}=\mathrm{O}$ str), 1657 (ketone $\mathrm{C}=\mathrm{O}$ str), 1370 (assym. S=O str), 1085 (symm. S=O str); ${ }^{1} \mathrm{H}$ NMR (400MHz, DMSO-d $)$ ): 7.59-7.57 (m, 2H, ArH), 7.30-7.24 (m, 4H, Ar-H), 7.15-7.12 (m, 4H, Ar-H), 6.79-6.77 (m, 2H, Ar-H), $6.32\left(\mathrm{~s}, 1 \mathrm{H}, \mathrm{H}_{5}\right)$, 3.92-3.87 (m, 3H, $\left.\mathrm{H}_{4}+\mathrm{OCH}_{2} \mathrm{CH}_{3}\right), 3.46-3.43\left(\mathrm{~m}, 1 \mathrm{H}, \mathrm{H}_{3}\right), 2.91-2.85\left(\mathrm{~m}, 2 \mathrm{H}, \mathrm{H}_{1}+\mathrm{H}_{2}\right), 2.26(\mathrm{~s}, 6 \mathrm{H}$, $\mathrm{CH}_{3}$ ), 0.93 (t, 3H, J 7.64 Hz, $\mathrm{OCH}_{2} \mathbf{C H}_{3}$ ); ${ }^{13} \mathrm{C}$ NMR (100 MHz, DMSO-d 6 ): 193.45, 169.67. $159.89,153.89,143.63,139.10,138.95,135.91,128.86,128.48,127.40,126.16,123.31,119.98$, $117.39,59.74,58.83,43.50,34.50,20.77,20.64,13.87$; MS (ESI): $m / z[\mathrm{M}+\mathrm{Na}]^{+}$calcd for $\mathrm{C}_{29} \mathrm{H}_{29} \mathrm{NNaO}_{5} \mathrm{~S}$ is 504.16, found 504.18; Anal. calcd for $\mathrm{C}_{29} \mathrm{H}_{29} \mathrm{NO}_{5} \mathrm{~S}$ (489.16): C, 69.16; H, 5.80; N, $2.78 \%$. Found: C, 69.23; H, 5.90; N, $2.91 \%$.

Ethyl 6-(3-chlorophenyl)-2-oxo-4-((4-(4-methylphenylsulfonamido)phenyl))-cyclohex-3-ene carboxylate (7p). Yellow solid, yield 91\%, 0.48, mp 240-241 ${ }^{\circ} \mathrm{C}$ (from EtOH), Rf 0.4 (30\% EtOAc:Hexane); IR $\left(v_{\max }, \mathrm{cm}^{-1}\right)$ : 1723 (ester $\mathrm{C}=\mathrm{O}$ str), 1635 (ketone $\mathrm{C}=\mathrm{O}$ str), 1369 (assym. $\mathrm{S}=\mathrm{O}$ str), 1078 (symm. $\mathrm{S}=\mathrm{O}$ str) cm ${ }^{-1} ;{ }^{1} \mathrm{H}$ NMR $\left(400 \mathrm{MHz}, \mathrm{DMSO}-d_{6}\right): 7.58-7.56$ (m, 2H, Ar-H), 7.50 (s,1H, Ar-H), 7.36-7.32 (m, 5H, Ar-H), 7.15-7.13 (m, 2H, Ar-H), 6.79-6.76 (m, 2H, Ar-H), 6.33 $\left(\mathrm{s}, 1 \mathrm{H}, \mathrm{H}_{5}\right), 4.01-3.98\left(\mathrm{~m}, 1 \mathrm{H}, \mathrm{H}_{4}\right), 3.92-3.90\left(\mathrm{~m}, 2 \mathrm{H}, \mathrm{OCH}_{2} \mathrm{CH}_{3}\right), 3.56-3.53\left(\mathrm{~m}, 1 \mathrm{H}, \mathrm{H}_{3}\right), 3.01$ $2.90\left(\mathrm{~m}, 2 \mathrm{H}, \mathrm{H}_{1}+\mathrm{H}_{2}\right), 2.27\left(\mathrm{~s}, 3 \mathrm{H}, \mathrm{CH}_{3}\right), 0.92\left(\mathrm{t}, 3 \mathrm{H}, J 6.84 \mathrm{~Hz}, \mathrm{OCH}_{2} \mathbf{C H}_{3}\right) ;{ }^{13} \mathrm{C} \mathrm{NMR}(100 \mathrm{MHz}$, DMSO- $\left.d_{6}\right)$ : $193.04,169.57,159.22,154.13,144.49,143.69,139.08,132.94,130.21,128.49$, 127.64, 126.46, 126.16, 123.08, 117.21, 59.86, 58.40, 38.88, 20.79, 13.86; MS (ESI): $\mathrm{m} / z$ [M+Na $]^{+}$ calcd for $\mathrm{C}_{28} \mathrm{H}_{26} \mathrm{ClNNaO}_{5} \mathrm{~S} 546.11$, observed 546.10; Anal. calcd for $\mathrm{C}_{28} \mathrm{H}_{26} \mathrm{ClNO}_{5} \mathrm{~S}$ (523.11): $\mathrm{C}$, 64.18; H, 5.00; N, 2.67; \%. Found: C, 64.08; H, 5.35; N, $2.76 \%$.

Ethyl 6-(4-chlorophenyl)-2-oxo-4-(4-methylsulfonamidophenyl)-cyclohex-3-ene carboxyl ate (7q). Yellow solid, yield 92\%, 0.41g, mp 181-182 ${ }^{\circ} \mathrm{C}$ (from EtOH), Rf 0.4 (30\% EtOAc:Hexane); IR $\left(v_{\max }, \mathrm{cm}^{-1}\right)$ : $3268(\mathrm{~N}-\mathrm{H}$ str), 1726 (ester $\mathrm{C}=\mathrm{O}$ str), 1652 (ketone $\mathrm{C}=\mathrm{O}$ str), 1334 (assym. $\mathrm{S}=\mathrm{O}$ str), 1155 (symm. S=O str) cm ${ }^{-1}$; ${ }^{1} \mathrm{H}$ NMR $\left(400 \mathrm{MHz}, \mathrm{DMSO}-d_{6}\right): 10.13(\mathrm{~s}, 1 \mathrm{H}, \mathrm{NH}), 7.76-7.71$ (m, 2H, Ar-H), 7.34-7.33 (m, 2H, Ar-H), 7.25-7.23 (m, 2H, Ar-H), 6.76 (s, 2H, Ar-H), 6.53 (s, 1H, $\left.\mathrm{H}_{5}\right), 4.16-4.12\left(\mathrm{~m}, 1 \mathrm{H}, \mathrm{H}_{4}\right), 3.97-3.96\left(\mathrm{~m}, 2 \mathrm{H}, \mathrm{OCH}_{2} \mathrm{CH}_{3}\right), 3.63-3.57\left(\mathrm{~m}, 3 \mathrm{H}, \mathrm{H}_{1}+\mathrm{H}_{2}+\mathrm{H}_{3}\right), 3.08$ (s, $3 \mathrm{H}, \mathrm{CH}_{3}$ ), 0.98 (t, 3H, J 7.2 Hz, $\left.\mathrm{OCH}_{2} \mathbf{C H}_{3}\right) ;{ }^{13} \mathrm{C}$ NMR (100 MHz, DMSO-d $)$ ): 194.24. 169.45, 158.44, 152.74, 140.74, 137.37, 136.33, 131.73, 127.93, 118.50, 104.96, 59.94, 58.61, 44.00, 35.00, 13.93; MS (ESI): $\mathrm{m} / z[\mathrm{M}+\mathrm{Na}]^{+}$calcd for $\mathrm{C}_{22} \mathrm{H}_{22} \mathrm{ClNNaO}_{5} \mathrm{~S}$ is 470.08, found 470.08; Anal. calcd for $\mathrm{C}_{22} \mathrm{H}_{22} \mathrm{ClNO}_{5} \mathrm{~S}$ (447.08): C, 58.99; H, 4.95; N, 3.13 \%. Found: C, 58.78; H, 4.83; N, 3.07 $\%$.

Ethyl 6-(3,4,5-trimethoxyphenyl)-2-oxo-4-(4-methylsulfonamidophenyl)-cyclohex-3-ene carboxylate (7r). Yellow solid, yield $84 \%, 0.42 \mathrm{~g}, \mathrm{mp} 194-195{ }^{\circ} \mathrm{C}$ (from EtOH), Rf 0.32 (30\% EtOAc:Hexane); IR $\left(v_{\max }, \mathrm{cm}^{-1}\right): 3208$ (N-H str), 1731 (ester $\mathrm{C}=\mathrm{O}$ str), 1640 (ketone $\mathrm{C}=\mathrm{O}$ str), 1331 (assym. $\mathrm{S}=\mathrm{O}$ str), 1144 (symm. $\mathrm{S}=\mathrm{O}$ str); ${ }^{1} \mathrm{H}$ NMR (400MHz, DMSO- $\left.d_{6}\right)$ : 10.13 (s, 1H, NH), 7.75 7.73 (m, 2H, Ar-H), 7.26-7.24 (m, 2H, Ar-H), 6.76 (s, 2H, Ar-H), 6.53 (s, 1H, H5), 4.16-4.12 (m, 
$\left.1 \mathrm{H}, \mathrm{H}_{4}\right), 3.97-3.96\left(\mathrm{~m}, 3 \mathrm{H}, \mathrm{OCH}_{2} \mathrm{CH}_{3}+\mathrm{H}_{3}\right), 3.76\left(\mathrm{~s}, 6 \mathrm{H}, \mathrm{OCH}_{3}\right), 3.63\left(\mathrm{~s}, 3 \mathrm{H}, \mathrm{OCH}_{3}\right), 3.59-3.57$ $\left(\mathrm{m}, 2 \mathrm{H}, \mathrm{H}_{1}+\mathrm{H}_{2}\right), 3.05$ (s, 3H, CH 3$), 0.98$ (t, 3H, J 7.2 Hz, $\left.\mathrm{OCH}_{2} \mathbf{C H}_{3}\right) ;{ }^{13} \mathrm{C}$ NMR (100, MHz, DMSO-d $)_{\text {) }} 193.2439,168.7763,159.3245,152.7751,140.7433,137.3793,136.2544,131.7323$, 127.3735, 121.5796, 118.0432, 104.5045, 61.4086, 60.4450, 59.9409, 58.5544, 55.8612, 44.0001, 35.079, 20.9553, 13.9312; MS (ESI): $\mathrm{m} / z$ [M+Na] $]^{+}$calcd for $\mathrm{C}_{22} \mathrm{H}_{29} \mathrm{ClNNaO}_{8} \mathrm{~S}$ is 526.15 found 526.16; Anal. calcd for $\mathrm{C}_{22} \mathrm{H}_{22} \mathrm{ClNO}_{5} \mathrm{~S}$ (503.16): C, 59.63; H, 5.80; N, 2.78 \%. Found: C, 59.40; H, 5.77; N, $2.65 \%$.

\section{Supporting Information}

Supporting information (Experimental details, ${ }^{1} \mathrm{H}$ and ${ }^{13} \mathrm{C}$ NMR spectra for the synthesized compounds), associated with this article can be found, in the online version.

\section{Acknowledgements}

This research is supported by UGC New Delhi, India (F. no. 41-335/ 2012 (SR) dt.13.07.2012). Authors are thankful to SIF, VIT University, Vellore for NMR spectral analysis.

\section{References}

1. Sheldon, R. A. Green Chem. 2005, 7, 267.

http://dx.doi.org/10.1039/b418069k

2. Tundo, P.; Anastas, P. T. Green chemistry: Challenging perspectives; Oxford University Press, USA, 2000.

3. Samajdar, S.; Becker, F. F.; Banik, B. K. Heterocycles 2001, 55, 1019.

http://dx.doi.org/10.3987/COM-01-9211

4. Balaji, B.; Sasidharan, M.; Kumar, R.; Chanda, B. Chem. Commun. 1996, 707. http://dx.doi.org/10.1039/CC9960000707

5. Reddy, M. V.; Kumar, P. C. R.; Reddy, G. C. S.; Reddy, C. S. C. R. Chim. 2014, 17, 1250. http://dx.doi.org/10.1016/j.crci.2014.01.026

6. Tamboli, A. H.; Chaugule, A. A.; Sheikh, F. A.; Chung, W.-J.; Kim, H. Chin. J. Catal. 2015, 36, 1365 .

http://dx.doi.org/10.1016/S1872-2067(15)60848-8

7. Hao, S.-H.; Zhang, X.-Y.; Dong, D.-Q.; Wang, Z.-L. Chin. Chem. Lett. 2015, 26, 599. http://dx.doi.org/10.1016/j.cclet.2014.12.018

8. Ameta, K. L. Res. Chem. Intermed. 2015, 41, 3433.

http://dx.doi.org/10.1007/s11164-013-1446-Z 
9. Mojtahedi, M.; Saidi, M.; Bolourtchian, M. J. Chem. Res. (S) 1999, 710.

10. Marquez, H.; Plutin, A.; Rodriguez, Y.; Perez, E.; Loupy, A. Synth. Commun. 2000, 30, 1067.

http://dx.doi.org/10.1080/00397910008087124

11. Khan, N. R.; Jadhav, S. V.; Rathod, V. K. Ultrason. Sonochem. 2015, 27, 522.

http://dx.doi.org/10.1016/j.ultsonch.2015.03.017

12. Lidström, P.; Tierney, J.; Wathey, B.; Westman, J. Tetrahedron 2001, 57, 9225. http://dx.doi.org/10.1016/S0040-4020(01)00906-1

13. Kim, J. H.; Ryu, H. W.; Shim, J. H.; Park, K. H.; Withers, S. G. ChemBioChem 2009, 10, 2475.

http://dx.doi.org/10.1002/cbic.200900108

14. Seo, W. D.; Kim, J. H.; Kang, J. E.; Ryu, H. W.; Curtis-Long, M. J.; Lee, H. S.; Yang, M. S.; Park, K. H. Bioorg. Med. Chem. Lett. 2005, 15, 5514.

http://dx.doi.org/10.1016/j.bmcl.2005.08.087

15. Moustafa, O. S.; Ahmad, R. A. Phosphorus, Sulfur Silicon Relat. Elem. 2003, 178, 475. http://dx.doi.org/10.1080/10426500307933

16. Chandak, H. S. Der Pharma Chem 2012, 4, 1054.

17. Nakayachi, T.; Yasumoto, E.; Nakano, K.; Morshed, S. R. M.; Hashimoto, K.; Kikuchi, H.; Nishikawa, H.; Kawase, M.; Sakagami, H. Anticancer Res. 2004, 24, 737.

18. Mori, K. Tetrahedron 1989, 45, 3233. http://dx.doi.org/10.1016/S0040-4020(01)81007-3

19. Plummer, E.; Stewart, T.; Byrne, K.; Pearce, G.; Silverstein, R. J. Chem. Ecol. 1976, 2, 307.

http://dx.doi.org/10.1007/BF00988280

20. Mori, K.; Tamada, S.; Uchida, M.; Mizumachi, N.; Tachibana, Y.; Matsui, M. Tetrahedron 1978, 34, 1901.

http://dx.doi.org/10.1016/0040-4020(78)80095-7

21. Boeckman, Jr., R. K.; del Rosario Rico Ferreira, M.; Mitchell, L. H.; Shao, P. J. Am. Chem. Soc. 2002, 124, 190.

http://dx.doi.org/10.1021/ja017194i

22. Modugu, N. R.; Mehta, G. Tetrahedron Lett. 2015, 56, 6030.

http://dx.doi.org/10.1016/j.tetlet.2015.09.043

23. Mac, D. H.; Samineni, R.; Sattar, A.; Chandrasekhar, S.; Yadav, J. S.; Grée, R. Tetrahedron 2011, 67, 9305.

http://dx.doi.org/10.1016/j.tet.2011.09.121

24. Horning, E.; Field, R. J. Am. Chem. Soc. 1946, 68, 384.

http://dx.doi.org/10.1021/ja01207a012

25. Pollini, G. P.; Benetti, S.; De Risi, C.; Zanirato, V. Tetrahedron 2010, 66, 2775.

http://dx.doi.org/10.1016/j.tet.2010.01.078 
26. Smith Jr, W. T.; Eftax, D. S. J. Org. Chem. 1956, 21, 174.

http://dx.doi.org/10.1021/jo01108a006

27. Martínez, R.; Mendoza, H. M.; Angeles, E. Synth. Commun. 1998, $28,2813$. http://dx.doi.org/10.1080/00397919808004857

28. Buzas, A.; Gagosz, F. J. Am. Chem. Soc. 2006, 128, 12614. http://dx.doi.org/10.1021/ja064223m

29. Okano, T.; Satou, Y.; Tamura, M.; Kiji, J. Bull. Chem. Soc. Jpn. 1997, 70, 1879. http://dx.doi.org/10.1246/bcsj.70.1879

30. Wang, F.; Liu, Y.; Qi, Z.; Dai, W.; Li, X. Tetrahedron Lett. 2014, 55, 6399. http://dx.doi.org/10.1016/j.tetlet.2014.09.093

31. Garcia-Raso, A.; Garcia-Raso, J.; Campaner, B.; Mestres, R.; Sinisterra, J. Synthesis 1982, 1982, 1037.

32. Iglesias, M.; Marinas, J.; Sinisterra, J. Tetrahedron 1987, 43, 2335.

http://dx.doi.org/10.1016/S0040-4020(01)86819-8

33. Qi, S.; Shi, K.; Gao, H.; Liu, Q.; Wang, H. Molecules 2007, 12, 988. http://dx.doi.org/10.3390/12050988

34. Shakil, N.; Singh, M. K.; Kumar, J.; Sathiyendiran, M.; Kumar, G.; Singh, M. K.; Pandey, R. P.; Pandey, A.; Parmar, V. J. Environ. Sci. Heal. B 2010, 45, 524. http://dx.doi.org/10.1080/03601234.2010.493482

35. ELSharief, A.; Ammar, Y.; Mohamed, Y.; Zaki, M. J. Indian Chem. Soc. 1984, 61, 537.

36. Jamode, V. S.; Chandak, H. S.; Bhagat, P. R. J. Indian Chem. Soc. 2008, 85, 1169.

37. Zeng, C. C.; Li, X. M.; Yan, H.; Zhong, R. G. Chin. J. Chem . 2007, 25, 1174. http://dx.doi.org/10.1002/cjoc.200790219

38. Hosseinzadeh, R.; Tajbakhsh, M.; Mohadjerani, M.; Alikarami, M. J. Chem. Sci. 2010, $122,143$.

http://dx.doi.org/10.1007/s12039-010-0015-x

39. Rosen, B. R.; Ruble, J. C.; Beauchamp, T. J.; Navarro, A. Org. Lett. 2011, 13, 2564. http://dx.doi.org/10.1021/ol200660s

40. Jamode, V. S.; Chandak, H. S.; Bhagat, P. R.; Tambekar, D. H. Indian J. Heterocycl. Chem. 2003, 12, 323.

41. Kim, E.-J.; Ryu, H. W.; Curtis-Long, M. J.; Han, J.; Kim, J. Y.; Cho, J. K.; Kang, D.; Park, K. H. Bioorg. Med. Chem. Lett. 2010, $20,4237$.

http://dx.doi.org/10.1016/j.bmcl.2010.05.033 